\title{
A star cluster at the edge of the Galaxy ${ }^{\star \star \star}$
}

\author{
J. Brand ${ }^{1}$ and J. G. A. Wouterloot ${ }^{2}$ \\ 1 INAF - Istituto di Radioastronomia, via P. Gobetti 101, 40129 Bologna, Italy \\ e-mail: brand@ira.inaf.it \\ 2 Joint Astronomy Centre, 660 N. A’ohoku Place, University Park, Hilo, HI 96720, USA \\ Received 14 April 2006 / Accepted 13 October 2006
}

ABSTRACT

\begin{abstract}
Context. This paper is part of our ongoing study of star formation in the (far-) outer Galaxy.
Aims. Our goal in this paper is to study stars and molecular gas in the direction of IRAS 06145+1455 (WB89-789). The kinematic distance of the associated molecular cloud is $11.9 \mathrm{kpc}$. With a galactocentric distance of $\sim 20.2 \mathrm{kpc}$, this object is at the edge of the (molecular) disk of the Galaxy.

Methods. We use near-IR $(J, H, K)$, molecular line-, and dust continuum observations.

Results. The near-IR data show the presence of an (embedded) cluster of about 60 stars, with a radius $\sim 1.3$ pc and an average stellar surface density $\sim 12 \mathrm{pc}^{-2}$. We find at least 14 stars with NIR-excess, 3 of which are possibly Class I objects. The cluster is embedded in a $\sim 1000 M_{\odot}$ molecular/dust core, from which a molecular outflow originates. The temperature of most of the outflowing gas is $\lesssim 40 \mathrm{~K}$, and the total mass of the swept-up material is $\lesssim 10 M_{\odot}$. Near the center of the flow, indications of much higher temperatures are found, probably due to shocks. A spectrum taken of one of the probable cluster members shows a tentative likeness to that of a K3 III-star (with an age of at least $20 \mathrm{Myr}$ ). If correct, this would confirm the kinematic distance.

Conclusions. This cluster is the furthest one from the Galactic center yet detected. The combination of old and recent activity implies that star formation has been going on for at least $20 \mathrm{Myr}$, which is difficult to understand considering the location of this object, where external triggers are either absent or weak, compared to the inner Galaxy. This suggests that once star formation is occurring, later generations of stars may form through the effect of the first generation of stars on the (remnants of) the original molecular cloud.
\end{abstract}

Key words. stars: formation - stars: pre-main sequence - ISM: clouds - ISM: individual objects: WB 89-789 (IRAS 06145+1455)

\section{Introduction}

The HI in our Galaxy extends out to galactocentric distances $R$ of at least 24-25 kpc (e.g., Wouterloot et al. 1990; McClure-Griffiths et al. 2004). From high-sensitivity $21-\mathrm{cm}$ observations, Knapp et al. (1978) found HI out to $R \approx 50 \mathrm{kpc}$ (for a flat rotation curve and $R_{0}=8.5 \mathrm{kpc}$ ). Molecular material and associated sites of star formation do not appear at such large distances, however. Star formation in the Galaxy has been observed to occur out to $R \approx 20 \mathrm{kpc}$ (e.g., Fich \& Blitz 1984; Wouterloot et al. 1988; Kobayashi \& Tokunaga 2000; Santos et al. 2000; Snell et al. 2002). Wouterloot \& Brand (1989) detected many molecular clouds (hence star formation sites) towards IRAS sources in the outer regions of the disk. Thus both star formation and star formation reservoirs (i.e., molecular clouds) are present at large distances from the Galactic center, but not as far out as the atomic hydrogen. This suggests that beyond about $R \approx 20 \mathrm{kpc}$, conditions are not favorable to transforming HI into $\mathrm{H}_{2}$ and then into stars. The cause of this is not known (in general the formation of $\mathrm{H}_{2}$ clouds from $\mathrm{HI}$ is not yet a settled manner, but it must have to do with the physical environment presented by the interstellar medium).

In the far-outer Galaxy ( $R>16 \mathrm{kpc}$; hereafter FOG) the physical environment differs from that in the inner Galaxy (for a summary see Brand \& Wouterloot 1995), the effects of which

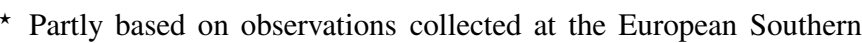
Observatory, Chile.

$\star \star$ Table 4 is only available in electronic form at http://www. aanda.org
}

may influence the formation of molecular clouds and the star formation process within them. Also, both the volume density of the molecular (and atomic) gas and the strength of spiral density waves are much reduced in the outer Galaxy. Even if star formation is independent of cloud formation and is to be initiated or enhanced by cloud-cloud collisions or triggered by supernovae or density waves, then star formation activity in the outer Galaxy would be expected to be significantly lower than in the inner Galaxy. To study the process of star formation and its end products in the outer parts of the galactic molecular disk, we observed a selection of FOG clouds in the NIR to directly detect the embedded stellar population. FOG clouds are at large enough $R$ for the physical conditions of the ISM to be different, yet those in the 2 nd and 3 rd quadrants are close enough for the star-forming cores to be resolved.

Here we present the most distant object studied with these observations: WB89-789 (IRAS 06145+1455; $\alpha_{2000}=$ $\left.06^{\mathrm{h}} 17^{\mathrm{m}} 24^{\mathrm{s}} 2, \delta_{2000}=+14^{\circ} 54^{\prime} 42^{\prime \prime}\right)$ at $\left(1, b=195^{\circ} .82,-0.57\right)$, which is located at $R \approx 20.2 \mathrm{kpc}, d \approx 11.9 \mathrm{kpc}$ (Brand \& Wouterloot 1994, hereafter BW94). The luminosity of the IRAS source $\left(L_{\mathrm{fir}} \approx 1.9 \times 10^{4} L_{\odot}\right)$ is consistent with a single star of type $\mathrm{B} 0.5 \mathrm{~V}$; it is embedded in a small (equivalent radius $r_{\mathrm{e}} \approx 5.7 \mathrm{pc}$, and $M \approx 5.6 \times 10^{3} M_{\odot}$ [BW94]) cloud. An $\mathrm{H}_{2} \mathrm{O}$ maser was detected towards this object (Wouterloot et al. 1993), indicating that this is an active star-forming region. No radio continuum emission was found within $4^{\prime}$ of the location of the IRAS source in our VLA A-array observations at $3.6 \mathrm{~cm}$ (Nov. 1993; unpublished). With a $4 \sigma$ detection limit of $\sim 3$ mJy we would have been able to detect an HII region ionized by a B0.5V star or earlier. 


\section{Observations and data reduction}

\subsection{Near-infrared}

The data presented here were obtained on February 15, 1995, with the ESO 2.2-m telescope at La Silla (Chile). Images in $J$, $H$, and $K$-bands were taken with the IRAC-2 camera and objective $C$, which resulted in a scale of $0.49 /$ pixel. The detector was a $256 \times 256$ pixel $^{2}$ NICMOS-III. The total instantaneous fieldof-view is therefore $\sim 2.1 \times 2.1 \operatorname{arcmin}^{2}$. During the observations the seeing was $0{ }^{\prime} \cdot 9$.

From a quick $K$-band exposure $(20 \times 3$ s) centered on the WB 89-789 IRAS point source position, a group of stars was seen to be small enough in extent to allow an observing procedure where it was placed at the center of each quadrant of the $\mathrm{CCD}$, as well as near the center of the CCD itself. Integrations in the $J_{-}, H$-, and $K$-bands were then made for $60 \times 2 \mathrm{~s}$, resulting in 5 separate images per band. After correction with a bad-pixel mask, each image was reduced separately by subtracting a starless sky image derived by median averaging from the other 4 exposures, and dividing by the flat field (see below). The reduced images were then averaged to get the final frame, which has an effective integration time of $10 \mathrm{~min}$. Immediately after these "ON-source" observations a comparison (OFF-) field was observed, at the same galactic latitude, but shifted towards smaller longitude by $0.2(\approx 42 \mathrm{pc}$ at the distance of WB89-789). To construct flat fields, dome exposures in all three bands were taken at the start and at the end of each night.

We observed 5 standard stars during the night, at different airmass. Each standard star was observed at 5 different positions on the CCD (with the same observing pattern as used for our object) in all 3 bands. Each observation was reduced individually and the derived photometric zero points (ZPs) were averaged, after which the ZP at the airmass of the WB89-789 observation was obtained through linear interpolation.

The standard star observations revealed a non-uniform CCD, with some quadrants "hotter" (i.e., registering more electrons for the same number of infalling photons) than others. This nonuniformity will increase the random error in the photometry of the science objects. In part this is accounted for by the average $\mathrm{ZP}$, as the standards were observed in the same way as the science object, hence the average ZP partly takes into account the differences between the quadrants.

The mosaiced images in $J, H$, and $K$ were aligned and truncated to show the same region of the sky. Stars were identified in each frame with the IRAF task DAOFIND, and photometry was performed through DAOPHOT, using a point-spread function determined from 5 relatively bright and isolated stars. Fitted stars were subtracted from the images, after which DAOFIND was run again on the residual frame. This procedure was repeated until no more stars were found (usually after 2 or 3 iterations). Derived magnitudes were then corrected to take into account the difference between the point spread fitting radius ( 3 pixels) and the aperture radius used (30 pixels) on the standard stars to derive the ZP; this correction amounts to $-0^{\mathrm{m}} .34$, -0.27 , and -0.24 for $J, H$, and $K$, respectively.

\subsection{Millimeter-lines}

\section{JCMT}

On January 24, 2003, we used the 15-m James Clerk Maxwell Telescope (JCMT) on Mauna Kea (Hawaii) to map the emission of ${ }^{12} \mathrm{CO}(2-1)$ towards WB89-789 in the raster (on-thefly) observing mode. The JCMT beam size at $230 \mathrm{GHz}$ is $22^{\prime \prime}$; observations were made on a $10^{\prime \prime}$ raster, using an autocorrelator spectrometer at a velocity resolution of $0.2 \mathrm{~km} \mathrm{~s}^{-1}$. An offposition was used at offset $600^{\prime \prime}$ in right ascension from the IRAS source position. The typical rms noise level in the spectra is $0.2 \mathrm{~K}\left(T_{\mathrm{A}}^{*}\right)$.

On August 7 and 8, 2003, the JCMT was used to simultaneously observe ${ }^{13} \mathrm{CO}(2-1)$ and $\mathrm{C}^{18} \mathrm{O}(2-1)$ emission in a $80^{\prime \prime} \times 80^{\prime \prime}$ region on a $10^{\prime \prime}$-raster towards WB89-789 using frequency-switching over $8.2 \mathrm{MHz}$. The typical rms noise level in these spectra is $0.13 \mathrm{~K}\left(T_{\mathrm{A}}^{*}\right)$. A comparison with separate (i.e., non-simultaneous) ${ }^{13} \mathrm{CO}(2-1)$ observations of line calibrators shows that our ${ }^{13} \mathrm{CO}(2-1)$ intensities must be corrected by multiplication with a factor of 0.88 .

On January 5, 2006, we used the JCMT to observe the ${ }^{12} \mathrm{CO}(3-2)$ transition towards WB89-789, in essentially the same region covered by the ${ }^{12} \mathrm{CO}(2-1)$ observations. Observations were made on a grid with 6" spacing; the beam size at $345.796 \mathrm{GHz}$ is $14^{\prime \prime}$. The velocity resolution is $0.136 \mathrm{~km} \mathrm{~s}^{-1}$, and typical rms noise level is $0.6 \mathrm{~K}\left(T_{\mathrm{A}}^{*}\right)$. Pointing was found to be accurate within 1 .' 8 . The line-data were reduced and analyzed with the programs CLASS and GRAPHIC, which are part of the GAG-software package developed by the Obs. de Grenoble and IRAM-Grenoble.

\section{IRAM 30-m}

WB89-789 was observed in CS $J=2-1,3-2$, and 5-4 with the IRAM 30-m telescope at Pico Veleta (Granada, Spain) in the period July 24-26, 1991. Maps were made of thirteen positions, with $15^{\prime \prime}$ spacing. The region mapped was sufficient to cover all CS emission. The telescope beamwidth at these frequencies $(98,147$, and $245 \mathrm{GHz})$ is respectively $26^{\prime \prime}, 16^{\prime \prime}$, and $11^{\prime \prime}$. All intensities are on a $T_{\mathrm{A}}^{*}$ scale. For CS(2-1) and (3-2) we used a $2 \times 128$-channel filterbank of $100 \mathrm{kHz}$ per channel, resulting in a resolution of 0.31 and $0.20 \mathrm{~km} \mathrm{~s}^{-1}$, respectively; CS(54) was observed with a resolution of $584 \mathrm{kHz}\left(0.71 \mathrm{~km} \mathrm{~s}^{-1}\right)$. Observations were made by position switching against an offposition $30^{\prime} \mathrm{W}$; the typical rms noise level in the spectra at the central position is $0.05 \mathrm{~K}$. All spectra were reduced with the Grenoble CLASS software.

\subsection{Millimeter-continuum}

\section{JCMT}

On April 10, 2002, we simultaneously observed the $450 \mu \mathrm{m}$ and $850 \mu \mathrm{m}$ emission towards WB89-789 with the Submillimetre Common-User Bolometer Array (SCUBA, Holland et al. 1999) at the JCMT. A standard 64-point jiggle map was made, which covers an area 2.3 in diameter; hence the observations are limited to the region immediately around the IRAS source. Calibration was performed by using fits to the time-dependence of skydips and the nearby Caltech Sub-millimeter Observatory (CSO) $225 \mathrm{GHz}$ optical depth data, and average ratios of $\tau(450 \mu \mathrm{m})$ and $\tau(850 \mu \mathrm{m})$ to $\tau(225 \mathrm{GHz})$. The final transition from instrumental parameters to Jy/beam was derived from similar maps of Mars. The half-power beam size at $450 \mu \mathrm{m}$ and $850 \mu \mathrm{m}$ are $9^{\prime \prime}$ and $15^{\prime \prime}$, respectively. The rms values in the WB89-789 maps are $450 \mathrm{mJy} /$ beam (at $450 \mu \mathrm{m}$ ) and $20 \mathrm{mJy} /$ beam (at $850 \mu \mathrm{m}$ ), respectively. The data were reduced with the program SURF (Jenness \& Lightfoot 1998). We found that away from the continuum source, the background emission level at both wavelengths was not around zero, but somewhat higher. We have corrected this by subtracting $1 \mathrm{Jy}$ and $0.05 \mathrm{Jy}$ from all pixel values in the $450 \mu \mathrm{m}$ and $850 \mu \mathrm{m}$ maps, respectively. 

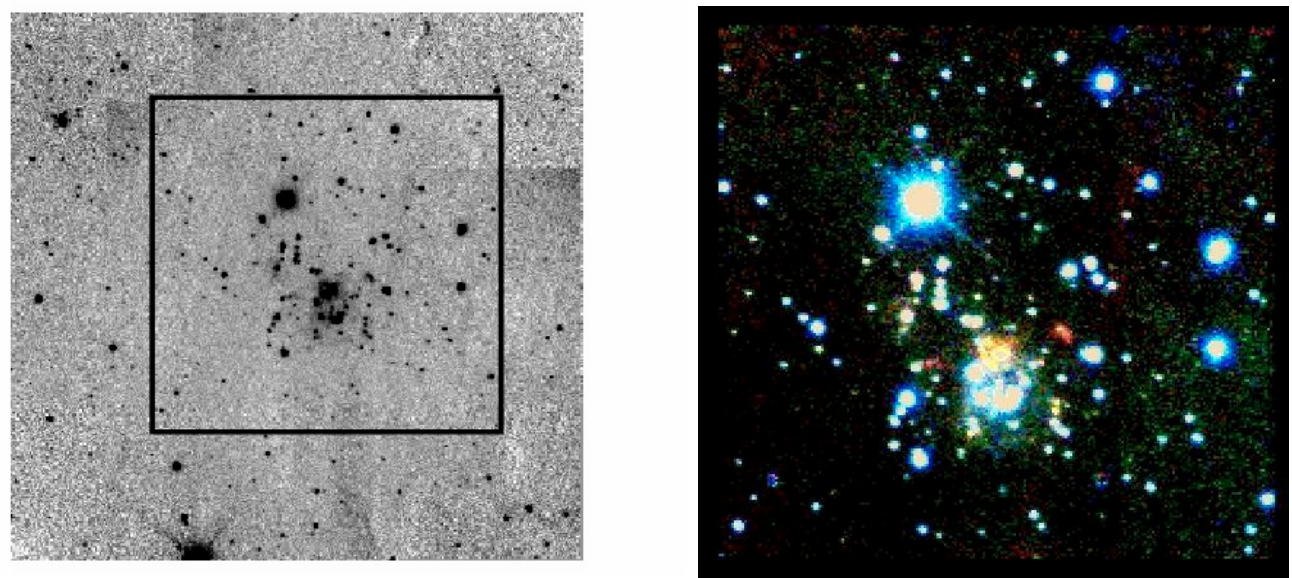

Fig. 1. (Left) a) $K$-band image of the region around WB89-789 (IRAS 06145+1455). The area of sky visible here is $\sim 3 \times 3 \mathrm{arcmin}^{2}$. The region outlined by the black box has a size of $\sim 1.7 \times 1.7 \mathrm{arcmin}^{2}$, and is shown on the right. North is up, East is left. (Right) b) False-color image ( $J=$ blue, $H=$ green, $K=$ red) of a $\sim 1.7 \times 1.7 \operatorname{arcmin}^{2}$ region around WB89-789.

\section{SEST}

Between August 17 and 22, 2003, the region around WB89-789 was observed in the 1.2-mm continuum with the 37-channel bolometer array SIMBA (SEST IMaging Bolometer Array) at the 15-m Swedish-ESO Submillimetre Telescope (SEST) at ESO-La Silla, Chile. A region of size $600^{\prime \prime} \times 600^{\prime \prime}$ (azimuth $\times$ elevation) was scanned at a rate of $80^{\prime \prime} / \mathrm{s}$; the total integration time per map was about $12 \mathrm{~min}$. Atmospheric opacity was determined from skydips, which were taken every $2 \mathrm{~h}$; values at the zenith ranged between 0.15 and 0.45 . The data were transformed from counts/beam to mJy/beam by making similar maps of Uranus once per day. The average conversion factor during this observing period is $58 \pm 6 \mathrm{mJy} /$ count.

In total, 8 maps were obtained. Each map was reduced and calibrated individually, after which they were averaged. The rms in the final image is $15 \mathrm{mJy} / \mathrm{beam}$. Pointing of the SEST was checked by observing a strong continuum source every $2 \mathrm{~h}$. The half-power beam width of the SEST at $1.2 \mathrm{~mm}$ is $23^{\prime \prime}$. Data were reduced with the program MOPSI, written by Robert Zylka (IRAM, Grenoble), following the instructions from the SIMBA Observers Handbook (Version 1.9, 9 Feb. 2003).

\subsection{Spectroscopy}

On December 7, 2004, we used the DOLORES spectrograph at the Telescopio Nazionale Galileo (TNG) at La Palma (in service observing-mode) to take a spectrum of one of the stars of the cluster. Six separate integrations of 2220 s each were taken using the high-resolution spectrograph and HR-B Grism No. 5 (dispersion $0.875 \AA$ per pixel), which resulted in a wavelength coverage of about 3200-4900 $\AA$, and (with a 1 " slit) in a spectral resolution of $\sim 3.2 \AA$. Bias frames were taken on the same night, and flat fields and $\mathrm{He}$ and Ar calibration-lamp spectra were taken the night before and after. As a flux calibrator, Hiltner600 was observed on the same night.

Data reduction was performed in IRAF, using standard packages. Bias frames were subtracted from all science and flat field frames. An average normalized flat field was constructed, which was corrected for bad pixels and then divided into the star frames. Wavelength calibration was performed with the Arlamp spectra, which in the wavelength range in question only has 8 useful (i.e., non-blended) lines, none below $3780 \AA$, and is therefore not very accurate.

\section{Results}

\subsection{Stars}

\subsubsection{Photometry, clustering, and pre-main sequence stars}

In Fig. 1a we show the $K$-band mosaic, while a false-color $(\mathrm{JHK})$ image of the area around WB89-789 (the region inside the box in Fig. 1a) is shown in Fig. 1b. Just below the center of the image we see a group of stars embedded in nebulosity; the IRAS source is located in the northern half of the nebulosity. In this $1.7 \times 1.7$ area DAOFIND detects 173,179 , and 161 stars in the $J$-, $H$-, and $K$-bands, respectively. The distributions of these stars in bins of 1 mag peak at $m_{J} \approx 20$ (faintest star found $19.96 \pm 0.24$ ), $m_{\mathrm{H}} \approx 18(19.59 \pm 0.54)$, and $m_{K} \approx 17$ (18.52 \pm 0.50$)$, respectively. Signal-to-noise ratios are reached of 5-10, i.e., photometric accuracy of $0.1-0.2$, at $m_{K}=16.5-17.5$, which corresponds to main sequence $[\mathrm{ms}]$ spectral types A0-5 (2-3 $\left.M_{\odot}\right)$ for a distance of $10 \mathrm{kpc}$ and visual extinction $A_{\mathrm{V}}=10 \mathrm{mag}$.

To confirm that WB89-789 is associated with a clustering of stars, in Fig. 2 we compare the density of stars in the WB89-789 field with that at a nearby off-position (see Sect. 2). Stars were counted in concentric rings of equal area

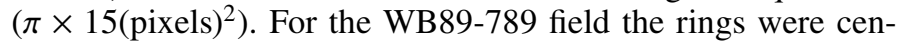
tered on the nebulous region visible in Fig. 3, and the counting was performed in the boxed-in area of the $K$-frame shown in Fig. 1a. There is a clear excess of stars compared to the background out to about $22^{\prime \prime}$ from the center of the nebulosity; this corresponds to a radius of $1.3 \mathrm{pc}$, in which the average stellar surface density is $12 \mathrm{pc}^{-2}$ (compared to $\leq 3 \mathrm{pc}^{-2}$ for the OFFfield). There is a particularly strong concentration of stars in the inner 15 pixels $\left(\sim 77^{\prime \prime} 5\right)$; in this region, with $r \sim 0.4 \mathrm{pc}$, the stellar surface density is $33 \mathrm{pc}^{-2}$.

The central 0'9 9 1.'1 part of the region shown in Fig. $1 \mathrm{~b}$ is where all 5 individual ON-source images overlap; a ( $K$-frame) contour plot of this region is shown in Fig. 3. Sixty-eight stars were detected in all three $(J, H, K)$ bands. The photometric data are collected in Table 4 . The $(J-H),(H-K)$-diagram of these stars is shown in Fig. 4. The dotted lines define the reddening band for normal stellar photospheres (Rieke \& Lebofsky 1985). Objects outside and to the right of this band have intrinsic NIR excess, and for a number of them their location in this diagram can be explained by the presence of circumstellar disks, with central holes of various dimensions (see Lada \& Adams 1992); these are likely pre-ms stars. 


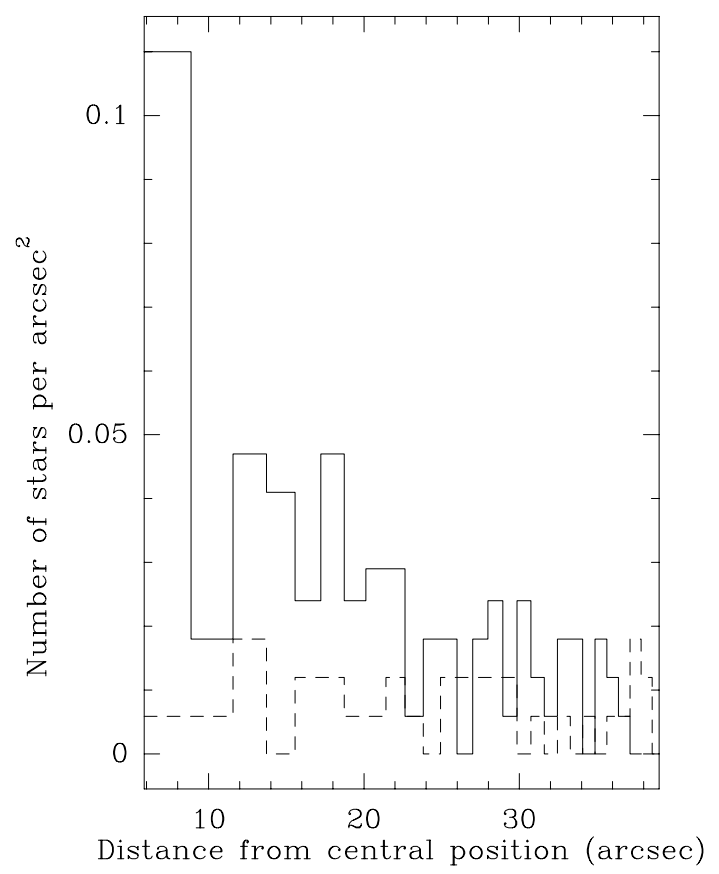

Fig. 2. Comparison between the star densities in the WB89-789 field (drawn) and in a field centered at a nearby off-position (dashed). Stars were counted on the $K$-images in concentric rings of equal area. In the WB89-789 field the rings were centered on the nebulosity (Fig. 1b). An excess of stars in the WB89-789 field is clearly visible.

There are 4 stars outside the normal reddening region on the left (Nos. 87, 53, 51, and 20). All of these except No. 87 are consistent with a location inside the normal region, considering error bars. On the other hand, stars with effective temperatures between $2500 \mathrm{~K}$ and $4000 \mathrm{~K}$ are predicted to have an excess of emission in the $\mathrm{H}$-band, which is due to the formation of $\mathrm{H}_{2}$ and absorption by $\mathrm{H}^{-}$(Gingerich \& Kumar 1964); this will shift stars like N. 87 to the left of the normal reddening region in Fig. 4 (see also Chini et al. 1992). There are 22 stars outside the normal region on the right. Eight (Nos. 7, 9, 13, 40, 63, 81, 98, 99) have anomalous colors, in that they are also below the unreddened T Tau locus; these are indicated with circles in Fig. 6. Three of these (Nos. 13, 40, 98) could be brought into normal region considering their error bars. The other 5 have "anomalous" colors, which could be due to unresolved binaries (Lada et al. 2000). That leaves at least 14 (22-8) stars with "true" NIR excess (i.e., $21 \%$ of the total of 68 ); these are indicated with a star in Fig. 6. Eleven are in the Class II-source region (between the right-hand normal reddening line and the right-most $\mathrm{T}$ Tau reddening line). Their SEDs are dominated by disk emission and their location can be explained by variation in size of central hole and inclination (see Lada \& Adams 1992). Three (No. 15, 54, 83) are in the region of Class I sources. These are the most embedded and youngest objects. Their SED is dominated by emission from an envelope of gas and dust.

\subsubsection{Extinction}

The amount of visual extinction towards WB89-789 is derived by means of a $K$ versus $(H-K)$ color-magnitude diagram (Fig. 5a), plotting only those stars that are within 7 '.5 from the center of the nebulosity and thus presumably part of the cluster. Here we can also use those (7) stars that were detected only in $H$ and $K$, but not in $J$. In addition to the stars, we have drawn the location of the unreddened main sequence for a distance of $11.9 \mathrm{kpc}$. Ignoring the stars that have been identified as having a NIR-excess in Fig. 4, because they do not follow the standard interstellar reddening law, we shift the main sequence along the direction of the interstellar reddening (indicated by the arrow in Fig. 5: $A_{K}=0.112 \times A_{\mathrm{V}}$ and $E(H-K)=0.061 \times A_{\mathrm{V}}$; Rieke $\&$ Lebofsky 1985) until it reaches the locations of the cluster stars. Thus we derive a maximum amount of foreground visual extinction of $A_{\mathrm{V}} \approx 6.25 \mathrm{mag}$ (maximum, because even the leastreddened stars can have some amount of internal extinction, i.e., due to the dust in the molecular cloud in which they are embedded). This may not seem much for such a supposedly distant object, but it is consistent with the findings of, e.g., Fich (1984) and Amôres \& Lépine (2005), who concluded that the extinction is fairly low ( $\sim 3 \mathrm{mag})$ in much of the outer Galaxy, and that there does not appear to be a progressively increasing extinction beyond a few kpc from the Sun.

In Fig. 5b we show all 68 stars for which we have $J, H$, and $K$, as well as the 15 stars for which only $H$ and $K$ are available. Again ignoring stars with NIR-excess (identified with a large open circle), as well as the stars for which we only have $H$ and $K$ (for which we cannot say whether they have NIR-excess) we see that there are stars with an additional extinction of up to $\sim 30 \mathrm{mag}$, although the bulk has $A_{\mathrm{V}} \lesssim 7.5$. This is about the same for stars inside and outside the above-defined cluster radius. As we mentioned in the Introduction, the edge of the molecular disk of the Galaxy lies at about $R \approx 20 \mathrm{kpc}$, thus it is unlikely that in our images of WB89-789 there are any stars more distant than the cluster, and we conclude that most stars shown are part of the cluster. Potentially the earliest-type star in the cluster that does not have NIR excess is star 33 (see Fig. 3): if one were to simply shift it back to the the main sequence, it would be of spectral type B0, consistent with the spectral type derived from the $L_{\mathrm{fir}}$ (and assuming it is an ms-star).

Figure 6 shows a section of the $K$-band image of the WB89-789 area, with the contours of integrated $\mathrm{C}^{18} \mathrm{O}(2-1)$ emission (see Fig. 7c) superimposed. The 14 stars with NIRexcess are shown in Fig. $4 \mathrm{~b}$, and the 8 stars with anomalous colors are marked in Fig. 6. Rather than being concentrated in the center of the cluster (which we take to be the nebulous region seen in Fig. 3), the NIR-excess stars are distributed in a ring around the peak of the $\mathrm{C}^{18} \mathrm{O}(2-1)$ emission. At the location of the molecular peak, no stars are seen in this $K$ image, which may be because the extinction there is too high. The average column density of $\mathrm{H}_{2}, N\left(\mathrm{H}_{2}\right)$, derived from the $\mathrm{C}^{18} \mathrm{O}(2-1)$ data (see Sect. 3.3.1) is $7.8 \times 10^{21} \mathrm{~cm}^{-2}$. Because $N\left(\mathrm{H}_{2}\right)=10^{21} \times A_{\mathrm{V}}$ (Bohlin et al. 1978, and assuming the gas-todust ratio in the FOG is the same as locally) this implies a visual extinction $A_{\mathrm{V}}$ of about 8 mag due to the whole of the molecular clump (front to back). At the peak of the $\mathrm{C}^{18} \mathrm{O}(2-1)$ emission $N\left(\mathrm{H}_{2}\right) \approx 1.0 \times 10^{22} \mathrm{~cm}^{-2}$, and thus $A_{\mathrm{V}} \approx 10 \mathrm{mag}$. If the cluster stars are embedded in the molecular gas, as they are likely to be, then the general (i.e., not counting that which is due to the circumstellar material) internal extinction would therefore be on average $\sim 5 \mathrm{mag}$ (and in any case $\leq 10 \mathrm{mag}$ ), consistent with the number derived above from the color-magnitude diagram.

\subsection{Molecular cloud}

\subsubsection{Cloud parameters}

\section{Whole cloud}

BW94 mapped the molecular cloud associated with WB89-789 in $\mathrm{CO}(1-0)$ with the SEST (beam size $45^{\prime \prime}$ ), on a $40^{\prime \prime}$ raster. 

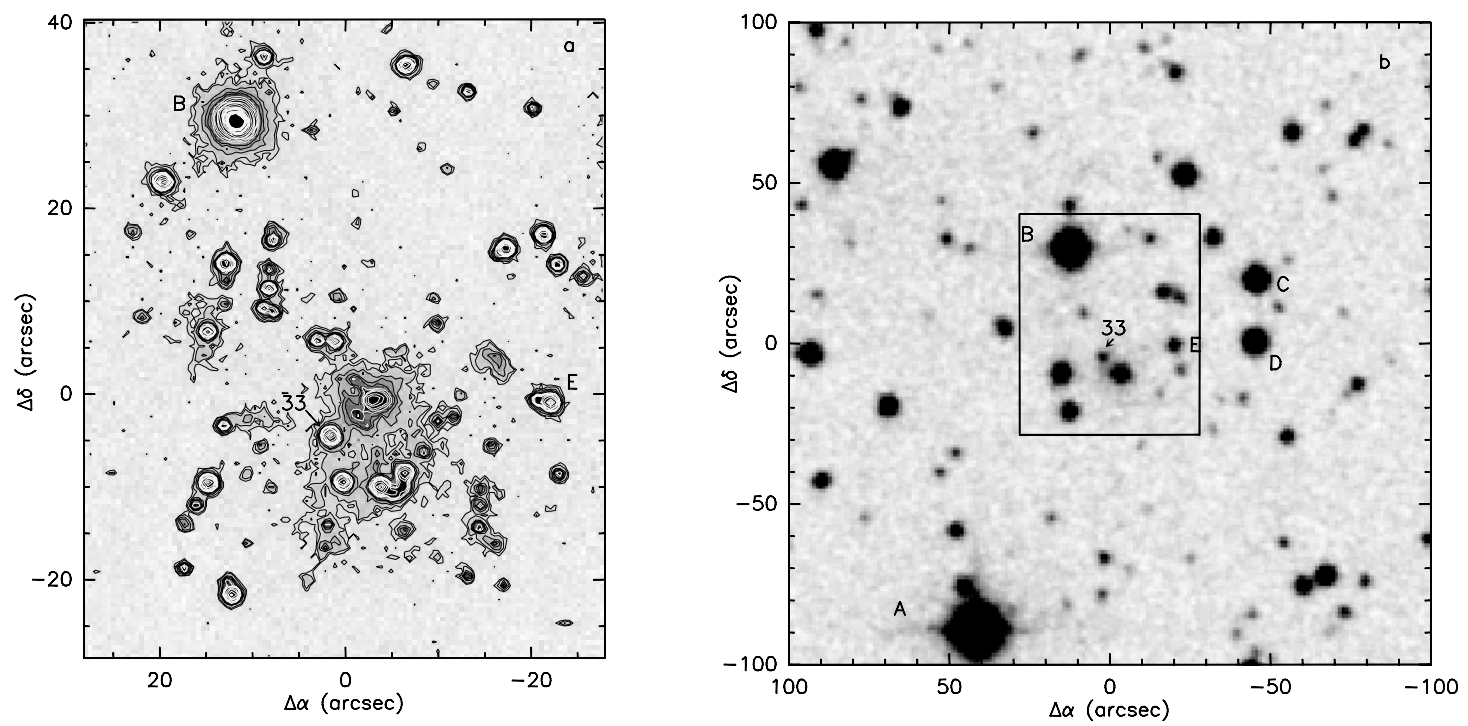

Fig. 3. a) Contour plot ( $K$-frame) of that part of the final mosaic where all $5 \mathrm{ON}$-source images overlap. Sixty-eight stars in this area have been detected in all three bands $(J, H$, and $K)$. Several stars are labeled, to identify the field in panel b). Among them is star 33, of which a spectrum has been taken (Sect. 2.4). North is up, East is left. The (0,0)-offset indicates the nominal position of the IRAS source. b) The cluster field from panel a) (inside the box) shown in a larger context on the POSS II-R image.
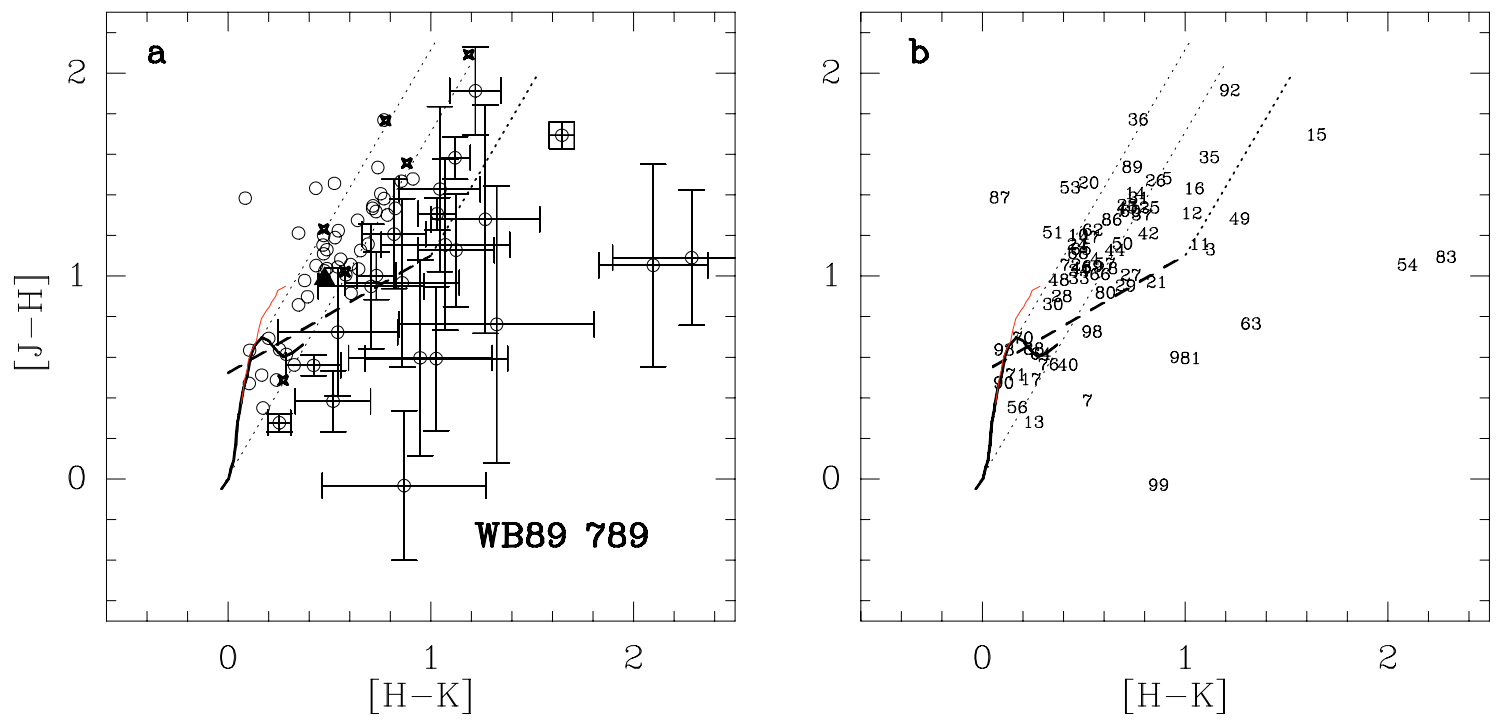

Fig. 4. a) NIR color-color diagram for the 68 stars in Fig. 3a that have reliable detections in all three $(J, H$, and $K)$ bands. The thick and thin drawn curves are the unreddened main sequence and giant branch, respectively, from Bessell \& Brett (1988); the dashed line indicates the loci of the classical T Tauri stars (Meyer et al. 1997); the dotted lines indicate the direction of normal interstellar reddening (Rieke \& Lebofsky 1985). Crosses on these lines mark increments of 5 mag of visual extinction from the points where they intersect the main sequence curve. Objects outside and to the right of the reddening band have intrinsic NIR excess; to avoid confusion, error bars are shown only for objects that are well outside the normal reddening band. The filled triangle identifies star 33 (see text and Fig. 3). b) Like a), but with the stars identified by their ID-number.

We have obtained new, better sampled $\mathrm{CO}(2-1)$ and (3-2) maps with the JCMT; the latter is shown in Fig. 7a. From the CO(2-1) data, following the procedures outlined in BW94, we derive a mass $M_{\mathrm{CO}} \approx 4.5 \times 10^{3} M_{\odot}\left(\right.$ from $N\left(\mathrm{H}_{2}\right)=X \int T_{\mathrm{R}}^{*}(\mathrm{CO}) \mathrm{d} v$, with $X=1.9 \times 10^{20} \mathrm{~cm}^{-2}\left(\mathrm{~K} \mathrm{~km} \mathrm{~s}^{-1}\right)^{-1}$ and using $T_{\mathrm{mb}}=\int T_{\mathrm{A}}^{*} / \eta_{\mathrm{fss}} \mathrm{d} v$; $\eta_{\mathrm{fss}}=0.80$ is the forward efficiency). The virial mass $M_{\mathrm{vir}} \approx$ $5.1 \times 10^{3} M_{\odot}\left(M_{\text {vir }}=126 r_{\mathrm{e}}(\Delta V)^{2}\right.$ for an $r^{-2}$ density distribution; MacLaren et al. 1988); $\Delta V$ is the FWHM line width, and the equivalent, beam-corrected radius $r_{\mathrm{e}} \approx 5.3 \mathrm{pc}$. These numbers agree very well with those derived by BW94 from the $\mathrm{CO}(1-0)$ map. We also note that the virial mass and the mass derived from the empirical method, using $X$, are in excellent agreement. The value of $X$ quoted above is the inner Galaxy value;
Brand \& Wouterloot (1995) have shown that in the outer Galaxy $X$ may be somewhat larger, but lies within $30-45 \%$ of the inner Galaxy value. Hence at the edge of the Galaxy, $X$ may be $2.8 \times 10^{20} \mathrm{~cm}^{-2}\left(\mathrm{~K} \mathrm{~km} \mathrm{~s}^{-1}\right)^{-1}$, which results in $M_{\mathrm{CO}} \approx$ 4.5-6.6 $\times 10^{3} M_{\odot}$. These results are collected in Table 1 .

\section{Cloud core: $\mathbf{C O}$}

In Fig. $7 \mathrm{~b}$ and $\mathrm{c}$ we show a plot of the integrated emission of ${ }^{13} \mathrm{CO}(2-1)$ and $\mathrm{C}^{18} \mathrm{O}(2-1)$, respectively. The emission in these two isotopomers was mapped only in the core of the cloud, but as can be seen from the maps, at least for $\mathrm{C}^{18} \mathrm{O}(2-1)$, all the emission is contained within the mapped region. While the ${ }^{13} \mathrm{CO}$ and ${ }^{12} \mathrm{CO}(2-1)$ emission peak at the IRAS position (offset $\left.0^{\prime \prime}, 0^{\prime \prime}\right)$, the $\mathrm{C}^{18} \mathrm{O}(2-1)$-peak is offset by about $10^{\prime \prime}$ to the 

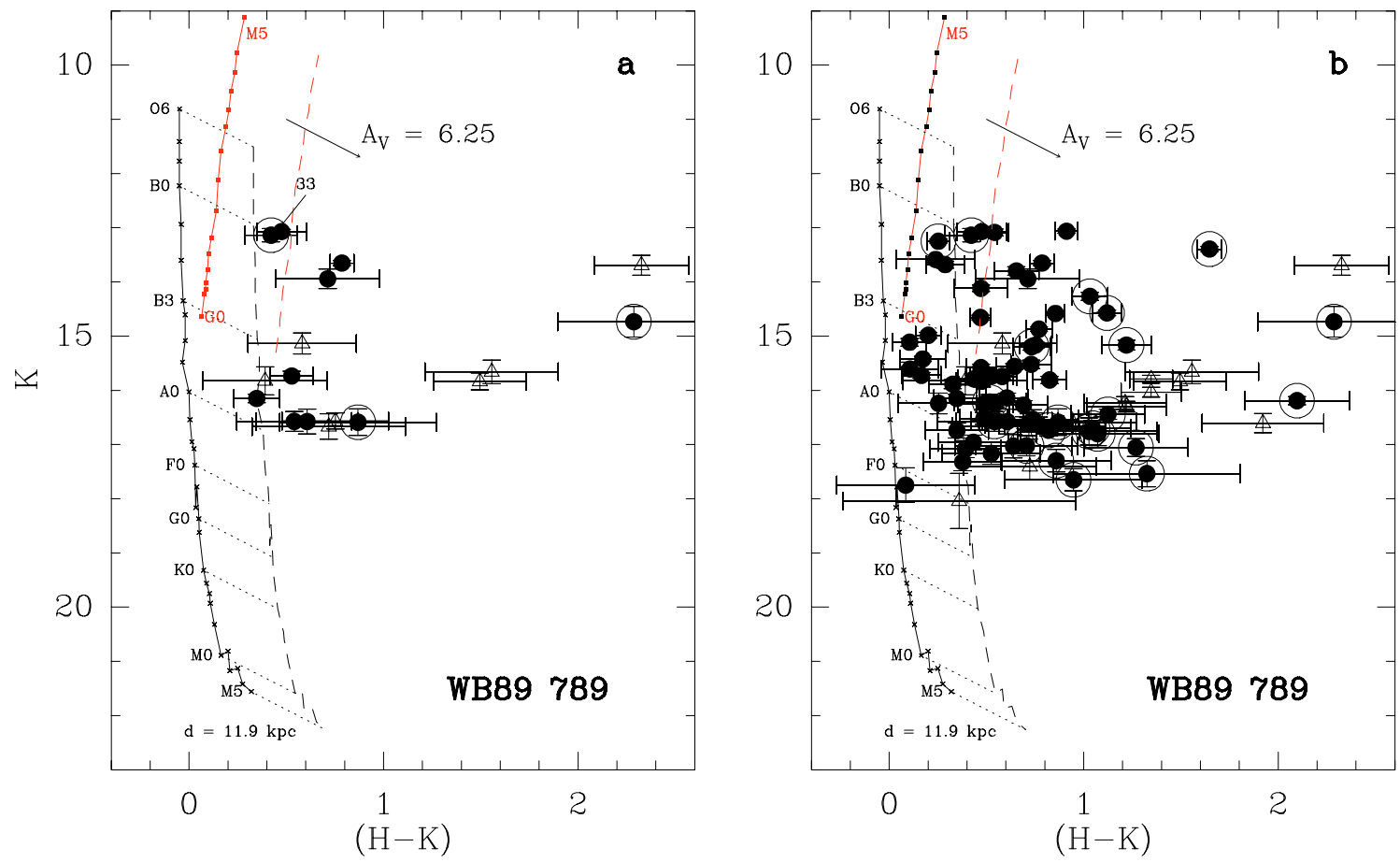

Fig. 5. $K$ versus $(H-K)$ color-magnitude diagram for WB89-789. a) Only stars within 15 pixels $(\sim 7.5)$ from the center of the nebula (Fig. $1 b)$. Filled circles are stars detected in all three bands $(J, H$, and $K)$; those with a NIR-excess have been additionally marked with an open circle. Open triangles: stars detected in $H$ and $K$ only. The drawn line marked O6-M5 is the unreddened main sequence for a distance of $11.9 \mathrm{kpc}$; data from Koornneef (1983, O6 - B7) and Bessell \& Brett (1988, B8 - M5). $K$-magnitudes were derived from $M_{V}\left(\right.$ Schmidt-Kaler 1982) and $(V-K)_{0}$. The dashed line is the main sequence for a distance of $11.9 \mathrm{kpc}$ and a visual extinction $A_{\mathrm{V}}=6.25 \mathrm{mag}$ (Rieke \& Lebofsky 1985). The drawn line marked G0-M5 is the unreddened giant branch (data from Bessell \& Brett 1988) for a distance of $11.9 \mathrm{kpc}$. Its location for $A_{\mathrm{V}}=6.25 \mathrm{mag}$ is shown as a dashed line parallel to the unreddened sequence. Star 33 (see text and Fig. 3) is indicated. b) Like a), but for all stars.

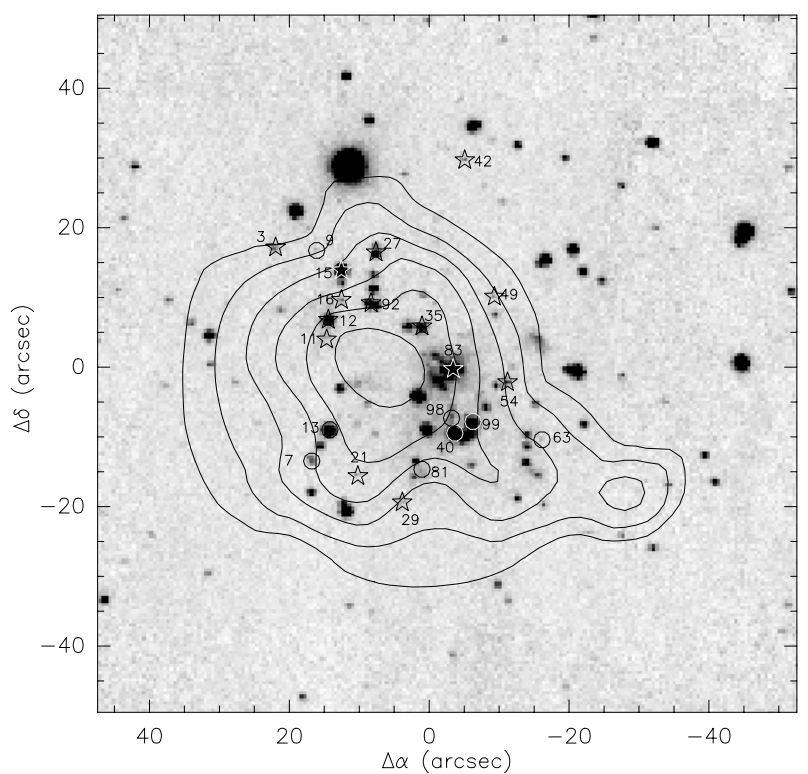

Fig. 6. $K$-band image of the WB89-789 region, with contours of the integrated $\mathrm{C}^{18} \mathrm{O}(2-1)$ emission superimposed (see Fig. 7c for details). The IRAS position is at $(0,0)$. The stars with NIR-excess are labeled, and identified with asterisks; those with anomalous colors (see text) are marked with circles.

East; this is not due to pointing uncertainties because ${ }^{13} \mathrm{CO}$ and $\mathrm{C}^{18} \mathrm{O}$ were observed simultaneously. The dust continuum emission (see Fig. 10) also peaks very close to the IRAS position; hence the offset detected in the $\mathrm{C}^{18} \mathrm{O}$ emission may be due to that molecule's depletion near the core in which the IRAS source is embedded (cf. Tafalla et al. 2004). The ${ }^{12} \mathrm{CO}$ emission, being more optically thick, only traces the outer layers of the core; also, ${ }^{13} \mathrm{CO}$ is more optically thick than $\mathrm{C}^{18} \mathrm{O}$ (see later) and traces the more outlying layers. The $\mathrm{C}^{18} \mathrm{O}(2-1)$ core is elongated in a SW-direction, similar to the ${ }^{12} \mathrm{CO}$ core in panel a. The coreradius derived from the ${ }^{13} \mathrm{CO}$ observations is identical to that, derived from ${ }^{12} \mathrm{CO}$, while the $\mathrm{C}^{18} \mathrm{O}$ core is $\sim 40 \%$ smaller. The masses $M_{\mathrm{CO}}$ and $M_{\mathrm{vir}}$ determined from ${ }^{12} \mathrm{CO}$ in the same region as mapped in ${ }^{13} \mathrm{CO}$ and $\mathrm{C}^{18} \mathrm{O}$ are reported in Cols. 4 and 6 , respectively, of Table 1 , under " $12 \mathrm{CO}$, core".

Column densities $N$ can be derived from $T_{\mathrm{mb}}\left({ }^{13} \mathrm{CO}\right)$ and $T_{\mathrm{mb}}\left(\mathrm{C}^{18} \mathrm{O}\right)$, assuming the emission is optically thin (see, e.g., Rohlfs \& Wilson 1996; Brand \& Wouterloot 1998, Eq. (1)). The excitation temperature $T_{\mathrm{ex}}$ is derived from the optically thick ${ }^{12} \mathrm{CO}$ transition. The ${ }^{12} \mathrm{CO}$ emission line is not just optically thick: the line profiles within $20-30^{\prime \prime}$ from the IRAS position show self-absorption. Examples are shown in Figs. 7d, e; we shall return to this in Sect. 3.2.2. From the emission at positions away from the region with self-absorption, we find that $T_{\mathrm{ex}} \sim 20 \mathrm{~K}$ is a representative value for this cloud. At each grid position of the cloud-map we derived $N\left({ }^{13} \mathrm{CO}\right)$ and $N\left(\mathrm{C}^{18} \mathrm{O}\right)$. Column densities of $\mathrm{H}_{2}$, and LTE-masses $M_{\text {tte }}$ were then derived through $N\left(\mathrm{H}_{2}\right) \approx 5 \times 10^{5} N\left({ }^{13} \mathrm{CO}\right)$ (Dickman \& Clemens 1983) and $N\left(\mathrm{H}_{2}\right) \approx 6 \times 10^{6} N\left(\mathrm{C}^{18} \mathrm{O}\right)$ (Frerking et al. 1982). The $M_{\text {lte }}$ are presented in Col. 5 of Table 1. From the detection equation, and the adopted value of $T_{\mathrm{ex}}$, we find that on average $\tau\left(\mathrm{C}^{18} \mathrm{O}\right) \sim 0.05$ (peak value 0.11 at offset $10^{\prime \prime}, 0^{\prime \prime}$ ), and $\tau\left({ }^{13} \mathrm{CO}\right) \sim 0.22$ (peak value 0.64 at offset $\left.0^{\prime \prime},-10^{\prime \prime}\right)$. It is clear that the $\mathrm{C}^{18} \mathrm{O}$ is optically thin, whereas the ${ }^{13} \mathrm{CO}$ is less so.

The $M_{\text {lte }}$ derived from ${ }^{13} \mathrm{CO}$ and $\mathrm{C}^{18} \mathrm{O}$ are in good agreement, but they are both smaller than the core-masses derived 

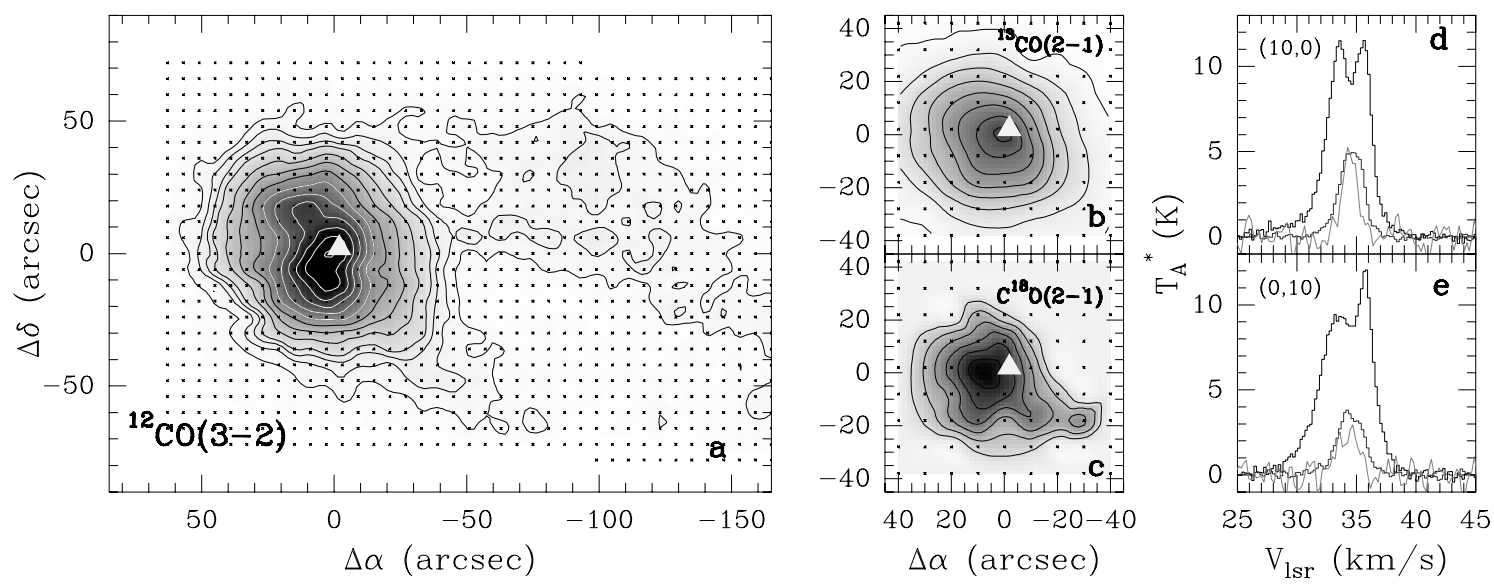

Fig. 7. JCMT-data. a) Map of the integrated intensity of $\mathrm{CO}(3-2)$ associated with WB89-789 $\left(\int T_{\mathrm{A}}^{*} \mathrm{~d} v\right.$; the integral is over the bulk of the emission, $30<V_{\mathrm{lsr}}<38 \mathrm{~km} \mathrm{~s}^{-1}$, and excludes the line wings). Contour levels $2.5(2.5) 10(5) 55 \mathrm{~K} \mathrm{~km} \mathrm{~s}^{-1}$ (lowest(step)highest). The filled triangle marks the peak of the SCUBA $850 \mu \mathrm{m}$ emission (at $-1{ }^{\prime \prime} .9,2{ }^{\prime \prime} 1$ ), and the observed positions are indicated with the tiny crosses. The IRAS point source position is at offset $(0,0)$. b) Map of the ${ }^{13} \mathrm{CO}(2-1)$ emission (area under the fitted Gaussians). Contour levels are $1(2) 15 \mathrm{~K} \mathrm{~km} \mathrm{~s}{ }^{-1}$. Triangle and crosses as in a). c) As b), but for the $\mathrm{C}^{18} \mathrm{O}(2-1)$ emission. Contour levels are $0.3(0.3) 2.1 \mathrm{~K} \mathrm{~km} \mathrm{~s}^{-1}$. d) Line profiles at offset position (10", $\left.0^{\prime \prime}\right)$. The strongest emission is the ${ }^{12} \mathrm{CO}(2-1)$-line; the profile clearly shows self-absorption. The less intense emission, plotted as a histogram, is the ${ }^{13} \mathrm{CO}(2-1)$ line, and the drawn line is that of $\mathrm{C}^{18} \mathrm{O}(2-1)$, multiplied by 4. e) Like d), but for offset position $\left(0^{\prime \prime}, 10^{\prime \prime}\right)$.

Table 1. Cloud parameters derived from the molecular line measurements.

\begin{tabular}{|c|c|c|c|c|c|c|}
\hline & $r_{\mathrm{e}}^{1}$ & $r_{\mathrm{hp}}^{1}$ & $\begin{array}{c}\Delta V \\
\mathrm{~km} \mathrm{~s}^{-1}\end{array}$ & $M_{\mathrm{CO}}^{2}$ & $\begin{array}{r}M_{\mathrm{lte}}^{3} \\
10^{3} M_{\odot}\end{array}$ & $M_{\mathrm{vir}}^{4}$ \\
\hline${ }^{12} \mathrm{CO}$, cloud & 5.2 & 1.6 & $\begin{array}{l}2.77^{5} \\
2.69^{5,6}\end{array}$ & $4.5-6.6$ & & $\begin{array}{l}5.1 \\
4.8^{6}\end{array}$ \\
\hline${ }^{12} \mathrm{CO}$, core & 2.9 & 1.6 & $\begin{array}{l}3.34^{5} \\
3.22^{5,6}\end{array}$ & $3.2-4.7$ & & $\begin{array}{l}4.1 \\
3.8^{6}\end{array}$ \\
\hline${ }^{13} \mathrm{CO}$, core & 2. & 1.1 & 2.14 & & $1.0-5.0$ & 1.6 \\
\hline $\mathrm{C}^{18} \mathrm{O}$, core & 1. & 0.9 & 1.72 & & $0.8-3.2$ & 0.6 \\
\hline CS, core & 1. & 0.7 & 2.07 & & & 0.4 \\
\hline
\end{tabular}

1. $r_{\mathrm{e}}$ : equivalent radius, corrected for beam size, at the $2-3 \sigma$-level; $r_{\mathrm{hp}}$ : the radius at half-maximum intensity level, corrected for beam size. 2. Via $N\left(\mathrm{H}_{2}\right)=X \int T_{\mathrm{mb}}(C O) \mathrm{d} v$. The indicated range of masses derives from using the inner Galaxy value $X=1.9 \times 10^{20} \mathrm{~cm}^{-2}\left(\mathrm{~K} \mathrm{~km} \mathrm{~s}^{-1}\right)^{-1}$ and allowing a possible $45 \%$ increase at the edge of the Galaxy (see text). 3. Assuming LTE-conditions, and $T_{\mathrm{ex}}=20 \mathrm{~K}$. Smaller values are for local abundance ratios for $\left[\mathrm{H}_{2}\right] /\left[{ }^{13} \mathrm{CO}\right]$ and $\left[\mathrm{H}_{2}\right] /\left[\mathrm{C}^{18} \mathrm{O}\right]$, higher values are for abundances extrapolated to the FOG (see text). 4. $M_{\text {vir }}=126 r$ $(\Delta V)^{2}$, for a density distribution $\propto r^{-2}$, and using $r_{\mathrm{e}}$. 5. Equivalent width, derived from the area under the emission line and the peak temperature. 6. Excluding the wing emission.

from ${ }^{12} \mathrm{CO}$. This is partly due to the fact that in ${ }^{12} \mathrm{CO}$ one sees the outer layers of the cloud, not seen in the other two isotopomers. In the derivation of the LTE-masses we have used the locally-determined abundance ratios $\left[\mathrm{H}_{2}\right] /\left[{ }^{13} \mathrm{CO}\right]$ and $\left[\mathrm{H}_{2}\right] /\left[\mathrm{C}^{18} \mathrm{O}\right]$. However, because of the galactic abundance gradients these values are certainly not valid at the edge of the Galaxy. As argued by Brand \& Wouterloot (1995), the ratio $\left[\mathrm{H}_{2}\right] /\left[{ }^{13} \mathrm{CO}\right]$ in the far-outer Galaxy might be about 5 times higher than locally. A similar extrapolation of the abundance gradients (Wilson \& Matteucci 1992; Wilson \& Rood 1994) suggests that the ratio $\left[\mathrm{H}_{2}\right] /\left[\mathrm{C}^{18} \mathrm{O}\right]$ in the far-outer Galaxy might be about 4 times higher than the local value. Taking this into account, $M_{\text {lte }}$ derived from ${ }^{13} \mathrm{CO}$ and $\mathrm{C}^{18} \mathrm{O}$ would become $5 \times 10^{3} M_{\odot}$ and $3.2 \times 10^{3} M_{\odot}$, respectively, which is closer to the range for $M_{\mathrm{CO}}$ (see Table 1).

\section{Cloud core: $\mathbf{C S}$}

All CS lines are detected essentially only at the IRAS point source position. The lines are Gaussian, showing no signs of outflow or other asymmetries. From the $\mathrm{CS}(2-1)$ map we find a (beam-corrected) radius of the CS-core of $0.7 \mathrm{pc}$, smaller even than the $\mathrm{C}^{18} \mathrm{O}$ core. With an average line width $\Delta V \approx$ $2.07 \mathrm{~km} \mathrm{~s}^{-1}$, we derive a virial mass of $M_{\mathrm{vir}} \approx 400 M_{\odot}$, similar to what was found from the $\mathrm{C}^{18} \mathrm{O}$ data. We have convolved the CS(3-2) and (5-4) spectra at the central position to the CS(21) beam, assuming Gaussian beam profiles. The resulting spectra were put on a main-beam brightness temperature scale $\left(T_{\mathrm{mb}}\right)$, and used in an LVG-model to derive density and kinetic temperature of the CS-gas. We ran the model with a range of parameters: $T=10-100 \mathrm{~K}$ in steps of $2 \mathrm{~K}, \log \left(n_{\mathrm{H}_{2}}\right)=4-6$ in steps of $0.1-$ 0.2 , and abundances $1 \times 10^{-11}, 3 \times 10^{-11}, 5 \times 10^{-11}$, and $1 \times 10^{-10}$. We used a velocity gradient $\mathrm{d} v / \mathrm{d} r \sim 1.5 \mathrm{~km} \mathrm{~s}^{-1} \mathrm{pc}^{-1}$. We found the smallest $\chi^{2}(\sim 0.6)$, hence the best reproduction of the observed line ratios, for a CS-abundance of $3 \times 10^{-11}, T_{\text {kin }} \approx 84 \mathrm{~K}$, and $\log \left(n_{\mathrm{H}_{2}} / \mathrm{cm}^{-3}\right) \approx 4.9$. The fit is not very sensitive to the temperature, as $\chi^{2}$ is within a factor of 2 of the minimum value over a range of about $30 \mathrm{~K}$. Nevertheless it is clear that the deeper layers of the core from which the CS emission originates are considerably hotter than the outer parts traced by ${ }^{12} \mathrm{CO}$.

\subsubsection{Molecular outflow}

The SEST CO(1-0) spectrum at the IRAS position showed a line profile with non-Gaussian wings (BW94). The new, higherresolution $\mathrm{CO}(2-1)$ data allow us to better outline the outflow (see Fig. 8a), which is completely contained within one SEST $\mathrm{CO}(1-0)$ beam ( 45"; Fig. 8 b). In Fig. 8a we show a positionvelocity diagram at $\Delta \delta=0^{\prime \prime}$. Enhanced emission in the line wings is clearly visible at $\Delta \alpha=0^{\prime \prime}$. Also clearly visible is a double-peak structure in the ${ }^{12} \mathrm{CO}(2-1)$ emission. At positions within $\sim 20^{\prime \prime}$ from the center of the map the lines show a significant indentation, as illustrated in Figs. 7d, e. By comparing the position-velocity map in Fig. 8a with the theoretical contour maps shown in Figs. 3 and 4 in Phillips et al. (1981), we conclude that the pattern seen in Fig. $8 \mathrm{a}$ is due to self-absorption (i.e., by a temperature gradient in the cloud core), rather than 

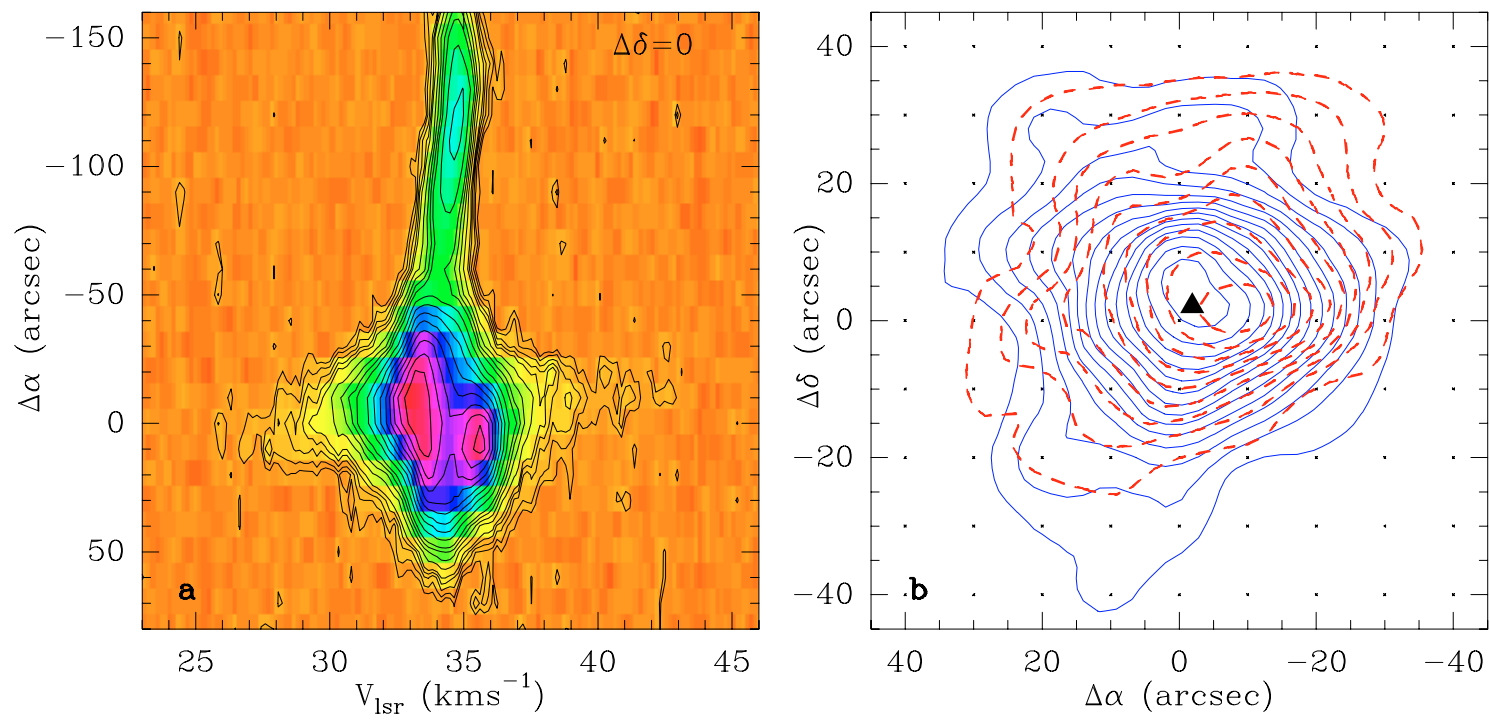

Fig. 8. a) $\Delta \alpha$ - velocity diagram at $\Delta \delta=0$ " of the ${ }^{12} \mathrm{CO}(2-1)$ emission. Contour levels are $0.25,0.4(0.2) 1(0.5) 3(1) 15 \mathrm{~K}$. At the location of the IRAS point source (at 0,0 ) emission in the wings of the line profiles is evident. b) Map of the integrated wing emission. Blue (drawn): $24.7<V_{\mathrm{lsr}}<31.7 \mathrm{~km} \mathrm{~s}^{-1}$; red (dashed): $36.7<V_{\mathrm{lsr}}<43.7 \mathrm{~km} \mathrm{~s}^{-1}$. Contour levels and step are $0.5 \mathrm{~K} \mathrm{~km} \mathrm{~s}^{-1}$. The filled triangle marks the position of the peak of the SCUBA $850 \mu \mathrm{m}$ emission $\left(-1{ }^{\prime \prime} 9,22^{\prime \prime} 1\right)$; the observed positions are indicated with the crosses.

Table 2. Parameters of the molecular outflow associated with WB89-789 ${ }^{\dagger}$.

\begin{tabular}{|c|c|c|c|c|c|c|c|c|c|c|c|c|c|c|c|c|c|c|}
\hline \multicolumn{19}{|c|}{$\overline{\mathrm{CO}(2-1)}$} \\
\hline Wing & \multicolumn{3}{|c|}{$\begin{array}{c}\text { Mass } \\
M_{\odot} \\
\end{array}$} & \multicolumn{3}{|c|}{$\begin{array}{c}\text { Mom. } \\
M_{\odot} \mathrm{km} \mathrm{s}^{-1} \\
\end{array}$} & \multicolumn{3}{|c|}{$\begin{array}{l}\text { Energy } \\
10^{44} \mathrm{erg}\end{array}$} & \multirow{2}{*}{$\begin{array}{c}\begin{array}{c}\text { Size } \\
\text { pc }\end{array} \\
1.4\end{array}$} & \multirow{2}{*}{$\frac{\begin{array}{c}V_{\text {out }} \\
\mathrm{km} \mathrm{s}^{-1}\end{array}}{3.5}$} & \multirow{2}{*}{$\frac{\begin{array}{c}t_{\mathrm{dyn}} \\
10^{5} \mathrm{yr}\end{array}}{4.0}$} & \multicolumn{3}{|c|}{$\begin{array}{c}F_{\mathrm{m}} \\
10^{-4} M_{\odot} \mathrm{km} \mathrm{s}^{-1} \mathrm{yr}^{-1}\end{array}$} & \multicolumn{3}{|c|}{$\begin{array}{c}L_{\mathrm{m}} \\
10^{-2} L_{\odot} \\
\end{array}$} \\
\hline blue & 5.1 & 3.2 & 4.1 & 18 & 11 & 15 & 6.7 & 4.2 & 5.4 & & & & 0.5 & 0.3 & 0.4 & 1.4 & 0.9 & 1.1 \\
\hline red & 4.0 & 2.6 & 3.3 & 14 & 9 & 11 & 4.7 & 3.0 & 3.8 & 1.6 & 3.3 & 4.8 & 0.3 & 0.2 & 0.2 & 0.8 & 0.5 & 0.7 \\
\hline \multicolumn{19}{|c|}{$\operatorname{CO}(3-2)$} \\
\hline blue & 6.4 & 4.0 & 3.4 & 28 & 18 & 15 & 15 & 10 & 8 & 1.1 & 4.4 & 2.4 & 1.2 & 0.7 & 0.6 & 5.2 & 3.3 & 2.8 \\
\hline red & 5.2 & 3.3 & 2.8 & 27 & 17 & 14 & 20 & 13 & 11 & 1.2 & 5.1 & 2.3 & 1.2 & 0.7 & 0.6 & 7.2 & 4.6 & 3.9 \\
\hline
\end{tabular}

$\dagger$ In case of three numbers per column, left: $T_{\mathrm{ex}}=20, \tau=1$, center: $T_{\mathrm{ex}}=20, \tau=0$, right: $T_{\mathrm{ex}}=40, \tau=0$. We used the local abundance ratio $[\mathrm{CO}] /\left[\mathrm{H}_{2}\right]=1.0 \times 10^{-4}$.

due to absorption by a colder (unrelated) foreground cloud. This is consistent with the fact that the CS-data reveal a higher temperature than the ${ }^{12} \mathrm{CO}$ (Sect. 3.2.1).

Again assuming LTE-conditions, we calculated the mass of the gas in the line wings from the ${ }^{12} \mathrm{CO}(2-1)$ data; the wings are not visible in the other two isotopomers. We adopted $T_{\mathrm{ex}}=20 \mathrm{~K}$, used $T_{\mathrm{mb}}$ (see Sect. 3.2.1), and considered both the optically thick and -thin cases. The total velocity intervals, 24.7-31.7 $\mathrm{km} \mathrm{s}^{-1}$ (blue wing) and 36.7-43.7 $\mathrm{km} \mathrm{s}^{-1}$ (red wing), over which was integrated (in $1 \mathrm{~km} \mathrm{~s}^{-1}$-wide bins), were chosen such that the emission of the quiescent gas was excluded.

In addition to the mass, we also calculated the flow's energy, momentum, size, and velocity (as outlined, e.g., by Lada 1985; Wouterloot \& Brand 1999); the dynamical age was then derived from the ratio of the flow's size $(\sim 3.5 \mathrm{pc})$ and its velocity. The outflow parameters are collected in Table 2. Here we also give the flow's mechanical luminosity $\left(L_{\mathrm{m}}=\right.$ Energy/age $)$ and the force needed to drive the flow $\left(F_{\mathrm{m}}=\right.$ Momentum/age $)$.

Our ${ }^{12} \mathrm{CO}(3-2)$ data, taken with a smaller beam size and smaller grid spacing, show self-absorbed profiles in the cloud core. Line wings are more extended than for $\mathrm{CO}(2-1)$, and reach $20 \mathrm{~km} \mathrm{~s}^{-1}$ (blue) and $55 \mathrm{~km} \mathrm{~s}^{-1}$ (red), respectively. The top panels of Fig. 9 show a position-velocity diagram at $\Delta \delta=0^{\prime \prime}$ (left), and a contour plot of the outflow lobes in the $\mathrm{CO}(3-2)$-emission (right).

We have convolved the $\mathrm{CO}(3-2)$ observations with a Gaussian beam to the resolution of the $\mathrm{CO}(2-1)$ data, and resampled all (2-1) and (3-2) spectra to a velocity resolution of $0.55 \mathrm{~km} \mathrm{~s}^{-1}$. Using $\eta_{\mathrm{fss}}=0.71,0.80$ for $\mathrm{CO}(3-2,2-1)$, we have determined the line ratio $R 32=T_{\mathrm{mb}}[\mathrm{CO}(3-2)] / T_{\mathrm{mb}}[\mathrm{CO}(2-1)]$ in each velocity channel where $T_{\mathrm{mb}}>1.5 \sigma$ for both lines. For optically thick lines the ratio $R 32$ tends to 1 for large values of $T_{\text {kin }}$, while the limit for optically thin lines is 2.25 (Bachiller \& Tafalla 1999; Codella et al. 2006). In the lower panels of Fig. 9 we show the results at two representative positions: near the center of the flow and the peak of the blue lobe (lower left) and at a more quiescent location in the cloud (lower right). Typical values for $R 32$ in the line center are $0.80-0.90$, corresponding to $T_{\text {kin }} \approx 15-30 \mathrm{~K}$, assuming both lines are optically thick; this is consistent with what we found from the $\mathrm{CO}(2-$ 1) lines, and from a fit to the spectral energy distribution (see Sect. 3.2.3). In the line wings, typically $R 32<1.5$, implying $T_{\text {kin }} \lesssim 40 \mathrm{~K}$ if both lines are optically thin away from the line center. But $R 32$ can reach values of 4 to 6 at some positions, as can be seen in the panel on the lower left in Fig. 9. This cannot be explained if both lines are optically thin or thick at the same time. On the other hand, the uncertainties in the high R32values are large, and assuming a more stringent criterion in their 

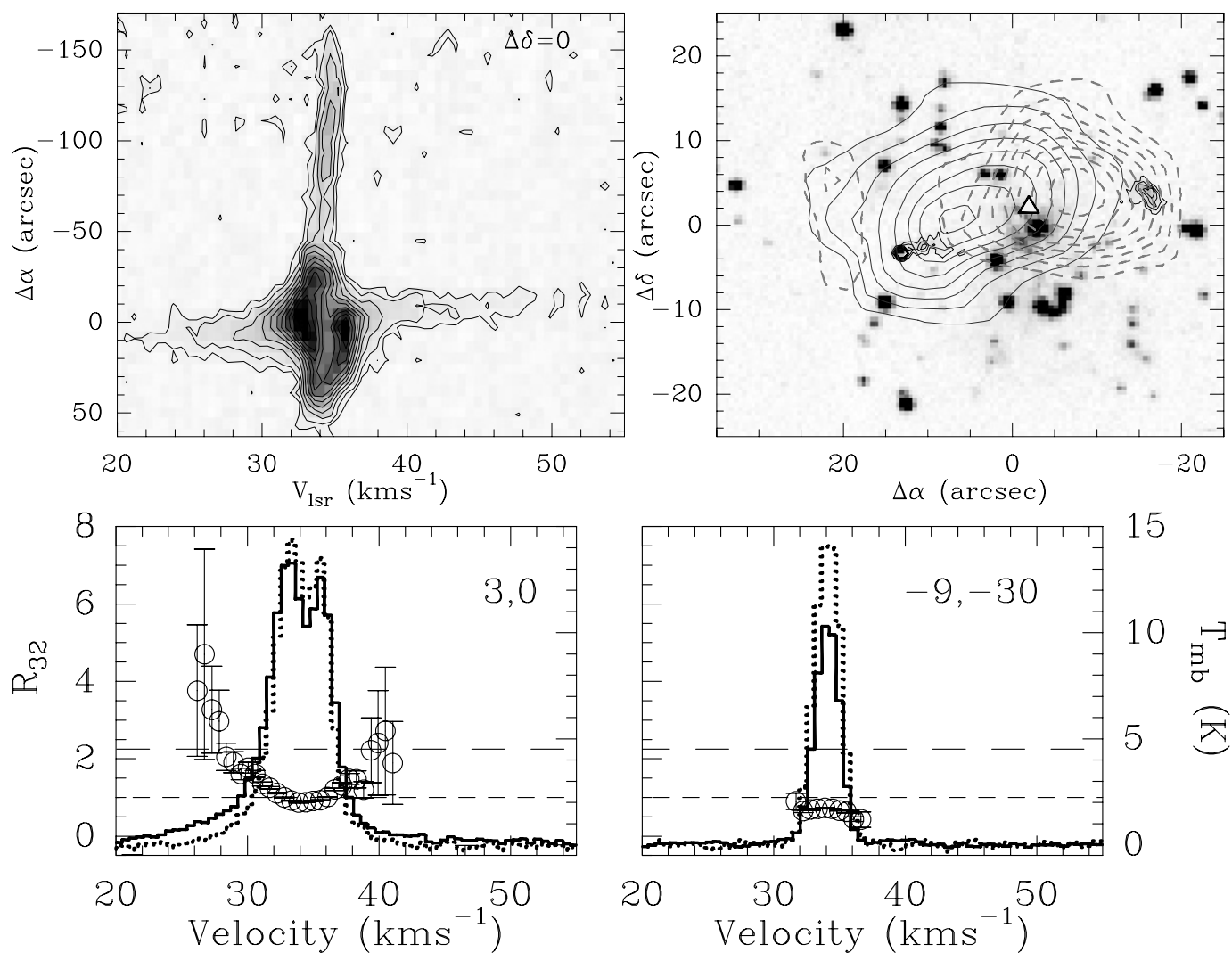

Fig. 9. Top (Left) $\Delta \alpha$ - velocity diagram at $\Delta \delta=0^{\prime \prime}$ of the ${ }^{12} \mathrm{CO}(3-2)$ emission. Contour levels are $0.5,1(1) 15 \mathrm{~K}$. (Right) Map of the integrated ${ }^{12} \mathrm{CO}(3-2)$ wing emission. Blue (drawn): $20<V_{\mathrm{lsr}}<31.2 \mathrm{~km} \mathrm{~s}^{-1}$; red (dashed): $37.2<V_{\mathrm{lsr}}<55 \mathrm{~km} \mathrm{~s}^{-1}$. Contour levels and step are $2.0 \mathrm{~K} \mathrm{~km} \mathrm{~s}{ }^{-1}$. The IRAS position is at $(0,0)$. The white triangle with black outline marks the position of the peak of the SCUBA $850 \mu \mathrm{m}$ emission. The underlying image is the $K$-frame of the region. Contours are drawn to highlight two extended features near offsets $\left(+10^{\prime \prime},-3^{\prime \prime}\right)$ and $\left(-16^{\prime \prime}, 4^{\prime \prime}\right)$ that might mark spots where the outflow impacts with the ambient medium. Bottom: Line profiles and ratios at two positions: near the center of the flow and the peak of the blue lobe (left) and in a quiescent part of the cloud (right). The offsets are shown inside the panels. The drawn line profile is that of $\mathrm{CO}(3-2)$, the dotted one if for $\mathrm{CO}(2-1)$; the temperature-scale is shown on the right-hand axis. The open circles with error bars indicate the line ratio $R 32$ for those velocity channels where $T_{\mathrm{mb}}>1.5 \sigma$ in both lines; values are shown on the left-hand axis. Short-dashed and long-dashed lines mark the upper limit of the line ratio if both lines are optically thick or thin, respectively (see text).

calculation, e.g., by requiring the value of $T_{\mathrm{mb}}$ to be at least $3 \sigma$ in both transitions, would obviously remove most (though not all) points with anomalous values of $R 32$. Ratios $R 32$ of around 2 are found for optically thin lines and $T_{\text {kin }} \approx 120 \mathrm{~K}$, which is not unexpected in the shocked gas in molecular outflows (see, e.g., Hatchell et al. 1999; Hirano \& Taniguchi 2001). A more accurate value of the temperature of the outflowing gas, and the possible existence of a gradient along the lobes, can be obtained only by observing higher transitions and at higher resolution (the JCMT beam sizes at the frequencies of the $\mathrm{CO}(3-2)$ and (2-1) transitions correspond to 0.8 and $1.2 \mathrm{pc}$, respectively).

Emission lines in the "tail" of the cloud, i.e., at offsets $\Delta \alpha \lesssim-60^{\prime \prime}$ (see Fig. 7) are less intense than in the core, with $T_{\mathrm{mb}} \leq 5 \mathrm{~K}$ and $\leq 7 \mathrm{~K}$ for $\mathrm{CO}(3-2)$ and (2-1), respectively. The lines are narrow $\left(\Delta V_{\text {fwhm }} \sim 1.1-1.5 \mathrm{~km} \mathrm{~s}^{-1}\right)$, becoming progressively narrower towards the $\mathrm{W}$-edge of the cloud. There is a slight velocity gradient in this part of the cloud, as can be seen in Figs. 8a and 9 (top, left). Line ratios $R 32$ indicate typical values of $T_{\text {kin }} \approx 8 \pm 2 \mathrm{~K}$ in this region.

Following the same procedure as outlined above, we have calculated various outflow parameters from the $\mathrm{CO}(3-2)$ data; these too are collected in Table 2 . The outflow in the $\operatorname{CO}(3-2)$ line is stronger and more extended in velocity, and more compact than that in the $\mathrm{CO}(2-1)$ line. The smaller size and higher average mass-weighted outflow velocity for the 3-2 flow result in a lower dynamical age. Other flow parameters are likewise influenced by this, but as can be seen from Table 2 , in general the results obtained from the two transitions are consistent within factors of a few. The lobes of this outflow have considerable overlap, indicating that the outflow axis is pointing close to the line-of-sight. As a consequence the size derived here is likely to be a lower limit, and age an upper limit. Values of $F_{\mathrm{m}}$ and $L_{\mathrm{m}}$ are thus lower limits too.

In the calculation of the flow parameters in Table 2 we have used the local abundance of $[\mathrm{CO}] /\left[\mathrm{H}_{2}\right]$. It is possible that the abundance ratio is lower at larger galactocentric distance. Gradients in $\mathrm{C} / \mathrm{H}$ and $\mathrm{O} / \mathrm{H}$ are similar (Wilson \& Matteucci 1992; Rolleston et al. 2000), and assuming that the $\mathrm{CO}$-abundance is determined by that in $\mathrm{C}$, one expects $[\mathrm{CO}] /\left[\mathrm{H}_{2}\right]$ to be a factor of about 4 lower in the far-outer Galaxy. This would increase all the parameters listed in Table 2 by a factor of 4, except for the size, flow velocity, and age. Keeping this in mind, a comparison with outflows associated with IR sources of similar luminosity (Beuther et al. 2002; Zhang et al. 2005) shows that this is not inconsistent with a typical outflow in an intermediate-mass star-forming region.

In Fig. 9, top right panel, we show the $\mathrm{CO}(3-2)$ outflow superimposed on the $K$-image. Near offsets $\left(+10^{\prime \prime},-3^{\prime \prime}\right)$ and $\left(-16^{\prime \prime}, 4^{\prime \prime}\right)$, bright extended spots are seen (cf. also Fig. 3a). The 

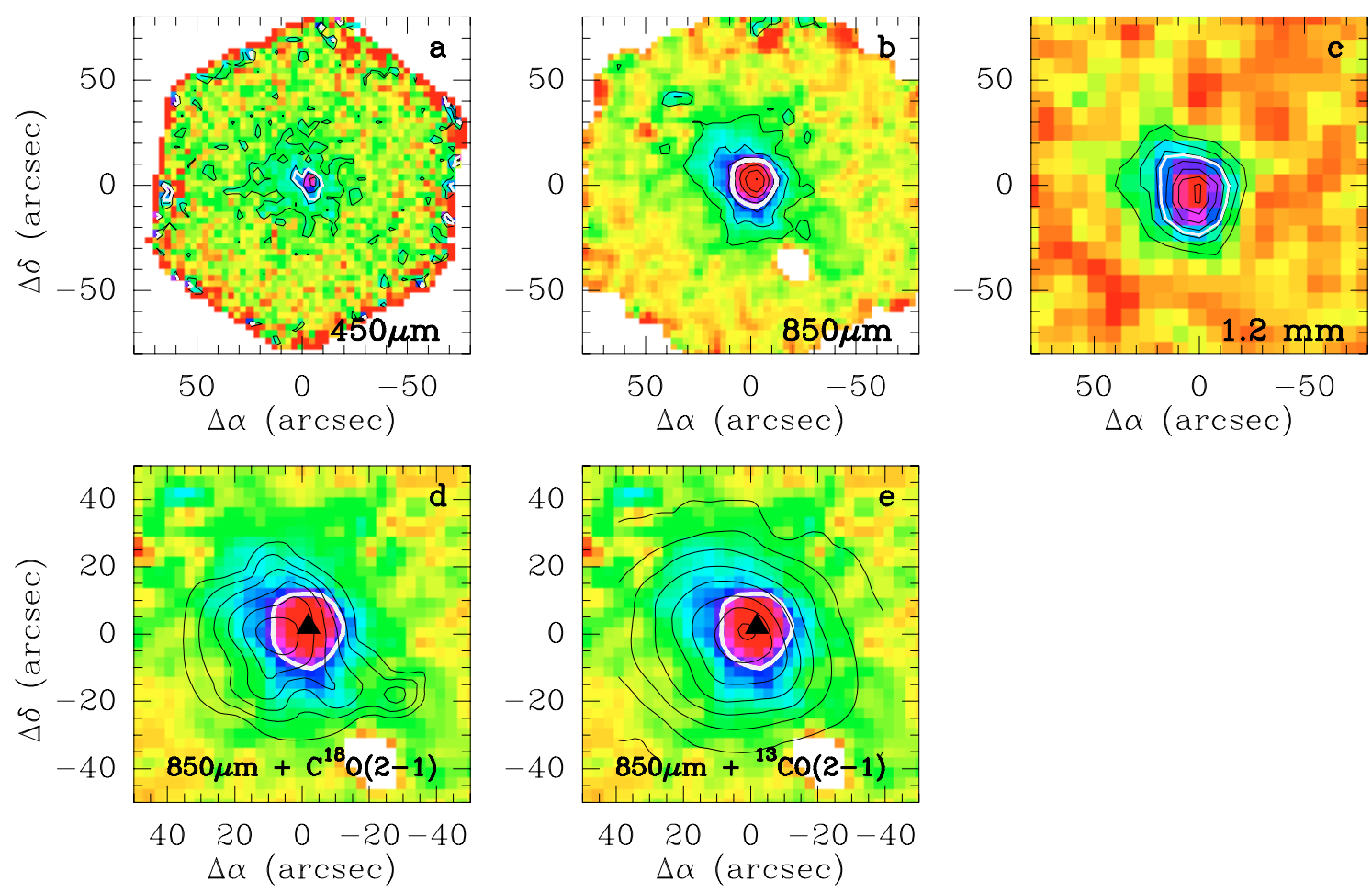

Fig. 10. Images of continuum emission at 450,850 , and $1200 \mu \mathrm{m}$, and overlays of the $850 \mu \mathrm{m}$ emission with the ${ }^{13} \mathrm{CO}$ and $\mathrm{C}^{18} \mathrm{O}$ emission. The IRAS position is at $(0,0)$. a) SCUBA $450 \mu \mathrm{m}$ map. Contour values $1(1) 5 \mathrm{Jy} /$ beam. The white contour indicates the half-peak level. b) SCUBA $850 \mu \mathrm{m}$ map. Contours $0.1(0.1) 0.7 \mathrm{Jy} / \mathrm{beam}$. White contour as in a). c) SIMBA $1.2 \mathrm{~mm}$ map. Contours $45(30) 230 \mathrm{mJy} / \mathrm{beam}$. White contour as in a). d) SCUBA $850 \mu \mathrm{m}$ map with $\mathrm{C}^{18} \mathrm{O}(2-1)$ contours superimposed. Contour values are $0.3(0.3) 2.1 \mathrm{~K} \mathrm{~km} \mathrm{~s}^{-1}$. The white contour indicates the $850 \mu \mathrm{m}$ emission half-peak level, while the triangle marks its peak. e) As d), but with ${ }^{13} \mathrm{CO}(2-1)$ contours superimposed. Contour values $1(2) 15 \mathrm{~K} \mathrm{~km} \mathrm{~s}^{-1}$. White contour and triangle as in $\left.\mathbf{d}\right)$.

Table 3. Cloud parameters derived from the continuum measurements.

\begin{tabular}{lcrrr}
\hline \hline & $r_{\mathrm{e}}^{1}$ & $r_{\mathrm{hp}}^{1}$ & \multicolumn{1}{c}{$\mathrm{S}^{2}$} & $M_{\text {dust }}^{3}$ \\
& \multicolumn{2}{c}{$\mathrm{pc}$} & \multicolumn{1}{c}{$\mathrm{Jy}$} & \multicolumn{1}{c}{$M_{\odot}$} \\
\hline $450 \mu \mathrm{m}$ & 0.85 & 0.25 & 12.9 & \\
$850 \mu \mathrm{m}$ & 1.48 & 0.39 & 1.8 & \\
$1.2 \mathrm{~mm}$ & 1.50 & 0.76 & 0.5 & \\
SED fit & & & & 11 \\
\hline
\end{tabular}

1. $r_{\mathrm{e}}, r_{\mathrm{hp}}$ : radius at $3 \sigma$-level and half-peak level, respectively; both corrected for beam size. 2. Integrated flux density above the $3 \sigma$-level. 3 . Assuming optically thin dust emission, a dust absorption coeff. $1 \mathrm{~cm}^{2} \mathrm{~g}^{-1}$ at $250 \mathrm{GHz}$; the exponent of the freq. dependence of the dust optical depth $\beta=1.8$, and $T_{\text {dust }}=23 \mathrm{~K}$ (see text for details).

spots are brightest in $K$, and virtually absent in $J$ (cf. Fig. 1b). This emission might be due to the $2.1 \mu \mathrm{m}$ line of $\mathrm{H}_{2}$, a manifestation of the interaction between outflow and ambient medium. This can be established only by taking a spectrum.

\subsubsection{Dust continuum}

In Fig. 10 we have collected the maps of the dust continuum, made at 3 different wavelengths, and shown their location with respect to the core found in the molecular lines. The ${ }^{13} \mathrm{CO}$ emission peaks very close to the maximum in the $850 \mu \mathrm{m}$ map, while the $\mathrm{C}^{18} \mathrm{O}$ peak is offset by about $10^{\prime \prime}$ ( 1 grid point $\sim$ half a beam width) to the East. Flux densities at the three wavelengths were calculated by integrating all emission above the $3 \sigma$-contour, and sizes of the dust-emission cores were determined from the half-peak value contour and were corrected for beam size. The results are collected in Table 3.

Figure 11 shows the spectral energy distribution (SED) of WB89-789. We have plotted our three FIR and mm-data points, in addition to the 4 IRAS flux densities, taken from the IPSC 2. We have made a grey-body fit to the data points for $100 \mu \mathrm{m}$ onwards; the fit is shown as the drawn curve. The 12,25 , and $60 \mu \mathrm{m}$ points cannot be fit by the same curve. As shown by Molinari et al. (1998) and Fontani et al. (2004) the flux densities at these wavelengths usually arise from regions outside the core that are detected at the longer wavelengths, and one needs at least 2 grey-body fits to cover the entire SED. The SED-fit shown in Fig. 11 was obtained by assuming a dust opacity $k_{v}=k_{250}(v[\mathrm{GHz}] / 250)^{\beta}$, with $k_{250}=1 \mathrm{~cm}^{2} \mathrm{~g}^{-1}$ (Ossenkopf $\&$ Henning 1981). We obtained a good fit with $\beta=1.8$, and a dust temperature $T_{\text {dust }}=23 \mathrm{~K}$. The resulting dust mass is $11 M_{\odot}$. For a gas-to-dust ratio of 100, the total mass of the core detected in the (sub-)mm continuum is $1100 M_{\odot}$, consistent with what is found from the ${ }^{13} \mathrm{CO}$ and $\mathrm{C}^{18} \mathrm{O}$-data (see Table 1). We should keep in mind, however, that the dust properties and the gas-todust ratio in the FOG may differ from those found near the Sun.

\section{The distance to WB89-789}

The presence of an outflow, together with the $\mathrm{H}_{2} \mathrm{O}$ maser emission detected by Wouterloot et al. (1993) demonstrates that star formation is still active in this object. If the kinematic distance of $11.9 \mathrm{kpc}$ can be confirmed, this would be the star-forming region furthest from the Galactic center yet found in the Galaxy. The distance was derived from the ${ }^{12} \mathrm{CO}$ velocity, using the Brand \& Blitz (1993) rotation curve. WB89-789 is at a galactic 


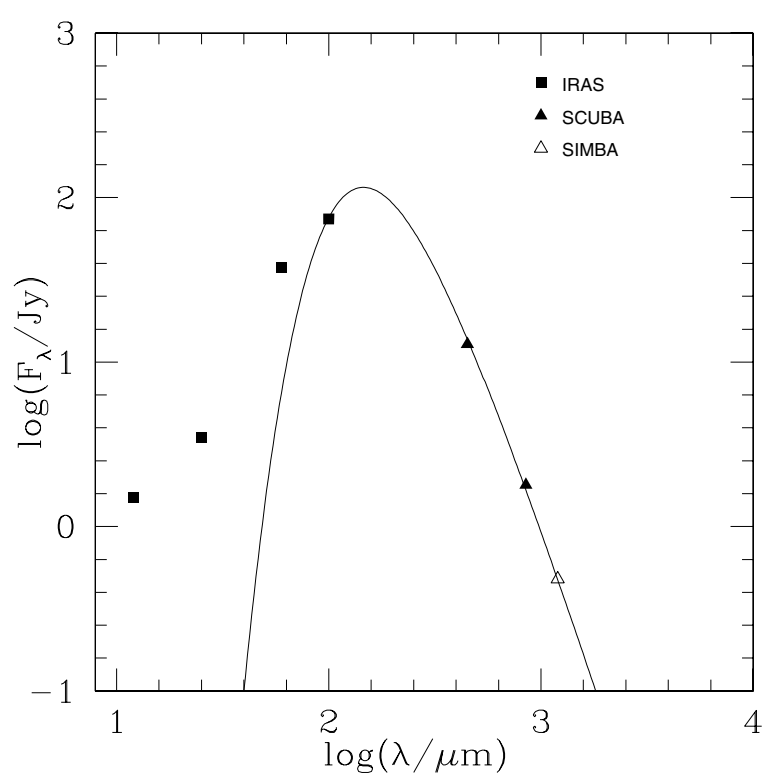

Fig. 11. Spectral energy distribution (SED) of WB89-789. Along the axes are $\log \lambda$ and $\log F$. The data shown are our own measurements of the continuum emission at 450,850 , and $1200 \mu \mathrm{m}$, together with the flux densities in the 4 IRAS bands (IPSC2). The curve shows the best fit to the data points at wavelengths $\geq 100 \mu \mathrm{m}$, which has $T_{\text {dust }}=23 \mathrm{~K}$, and a dust absorption coefficient of 1.8 , and which was obtained by assuming a dust opacity at $250 \mathrm{GHz}$ of $1 \mathrm{~cm}^{2} \mathrm{~g}^{-1}$.

longitude of $195^{\circ} 82$; the determination of kinematic distances is notoriously uncertain in directions near the (anti-) center, where $V_{\text {lsr }}$ is not very sensitive to distance, and random motions become relatively influential in the distance determination. If we assume a cloud-cloud velocity dispersion of $\sim 5 \mathrm{~km} \mathrm{~s}^{-1}$ (see Brand \& Blitz 1993), then the range of galactocentric distance of WB89-789 is $17 \lesssim R \lesssim 25 \mathrm{kpc}$ (with a corresponding range in heliocentric distance $8.6 \lesssim d \lesssim 17.1 \mathrm{kpc}$ ).

As shown in Fig. 5a, star 33 (indicated in Fig. 3), if it is a main sequence star, would be its earliest type member that does not have NIR excess, and would have a spectral type $\sim \mathrm{B} 0$, if the cluster is at a distance of $11.9 \mathrm{kpc}$. To check this, we have obtained a spectrum of this star (see Fig. 12). From the way the continuum rises towards longer wavelengths it is clear that star 33 is not an early-type star. We have compared the TNG-spectrum with those in the library of Jacoby et al. (1984). To reduce the noise we smoothed the spectrum to a resolution of $5.4 \AA$, comparable to the $4.5 \AA$-resolution of the Jacoby et al. (1984) spectra. From a visual inspection we conclude that the spectrum of star 33 agrees best with that of a K3 III-star (Jacoby et al. 1984, spectrum No. 100; this spectrum is also shown in Fig. 12). This implies that star 33 has a visual extinction $A_{\mathrm{V}} \sim 5.5$, and a geocentric distance of $10.7 \mathrm{kpc}$, i.e., $R \approx 19.0 \mathrm{kpc}$. Identification as a K3 III-star is consistent with the location of star 33 in the color-magnitude diagram of Fig. 5: dereddening brings it first on the giant branch, at around spectral type K3.

This result is rather surprising: star 33 is embedded in nebulosity (cf. Fig. 3a), making it a likely member of the cluster associated with the IRAS source. On the other hand, a K3III-star would originate from a 9-10 $M_{\odot}$ ms-star (i.e., spectral type B2B3), and has an age of about 20-25 Myr. It is possible that star formation has been going on in this region for a much longer time, and that the IRAS source, the water maser, the outflow, and the NIR-excess stars are just the latest episode. The earlier

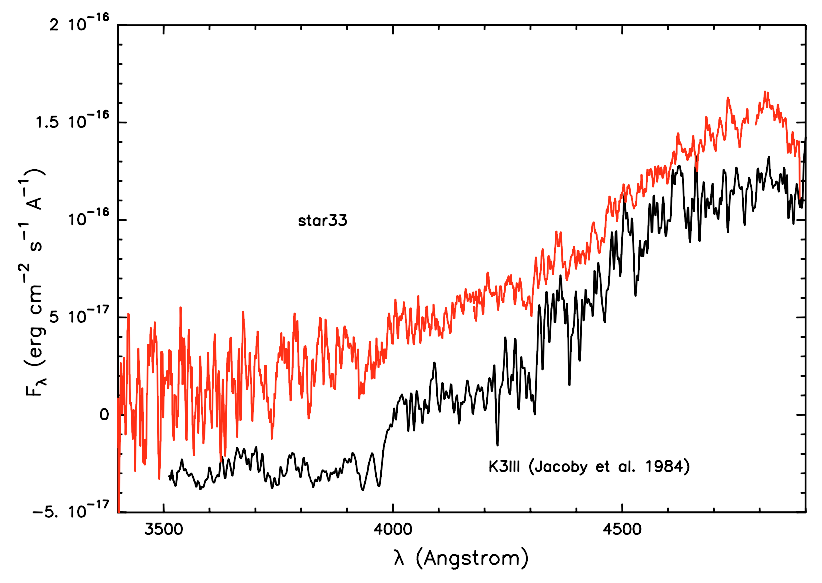

Fig. 12. Spectrum of star 33 (see Fig. 3) taken with DOLORES at the TNG. The spectrum has been smoothed to a resolution of $\sim 5.4 \AA$. Also shown is the spectrum of a K3III-star (from Jacoby et al. 1984, arbitrarily shifted vertically to allow comparison). Because of the poor wavelength calibration, the spectrum of star 33 has been shifted in $\lambda$ to get correspondence between the features in both stars. The amount of shifting necessary agrees with that which we determined from a comparison of the lines in our flux calibrator (Hiltner 600) and Jacoby et al. (1984) star No. 13, which has a similar spectral type.

occurrence of star formation (giving rise to star 33) would have removed much of the original molecular cloud, perhaps explaining why the cloud in which star formation is currently taking place is so small.

If this scenario is correct, then it still has to be explained how star formation can be sustained over such a long time, especially in this part of the Galaxy. As mentioned in the Introduction, if star formation is to be initiated by external triggers, then it is difficult to see how this can work efficiently in the far-outer Galaxy, where external triggers (SN explosions, spiral arms) are much more scarce or weak. On the other hand, as already found by Wouterloot et al. (1988) and Snell et al. (2002), once star formation does occur in FOG-clouds, it appears to proceed as it does locally. It should also be pointed out that there are other examples of older stars located in younger clusters. Kumar et al. (2004) found three late-type giants in two young (1.5-1.9 Myr) open clusters, indicating that star formation has been going on there for about 25 Myr. However, the spectral classification of star 33 is very tentative, and further speculation should wait until a better spectrum is available.

\section{Conclusions}

We have studied the star-forming region associated with IRAS 06145+1455 (WB89-789) in detail. Its location near the edge of the molecular disk (at a galactocentric distance of $20.2 \mathrm{kpc}$ ) makes this the galactic star forming region furthest from the Galactic center found up to now. An embedded cluster containing about 60 stars was found in $J H K$-images. The radius of the cluster is about $1.3 \mathrm{pc}$, within which the average stellar surface density is $12 \mathrm{pc}^{-2}$; within a core radius of $0.4 \mathrm{pc}$ the stellar surface density is $33 \mathrm{pc}^{-2}$. The stars have a foreground extinction of about 6 mag and an internal extinction of up to 30 mag, though typically $\sim 7.5 \mathrm{mag}$. At least 14 of the embedded stars show NIR-color excess in a $J-H$ vs. $H-K$ diagram, suggestive of the presence of disks around these stars; three of these are possibly of Class I, while the locations of the others suggest they are Class II objects. 
An optical spectrum of what seemed like it could be the earliest type cluster member with normal extinction shows a tentative likeness to that of a K3 III star. The distance of this star would then be $10.7 \mathrm{kpc}$ (galactocentric distance $19.0 \mathrm{kpc}$ ), consistent with the kinematical distance of the region. The presence of this star in the cluster suggests that star formation has proceeded for the past several $10^{7}$ years. The absence of radio continuum emission towards this source, at the sensitivity of our observations, indicates that no star of type B0.5 V or earlier is present.

JCMT ${ }^{13} \mathrm{CO}$ and $\mathrm{C}^{18} \mathrm{O}$ and IRAM CS observations show that the cluster is embedded in a $\sim 500-1500 M_{\odot}\left(M_{\text {vir }}\right)$ core. A JCMT ${ }^{12} \mathrm{CO}$ map shows that the core is contained in a $\sim 5000 M_{\odot}$ cloud, consisting of two clumps. The clump with the embedded cluster shows the existence of moderate outflow emission. (Sub-)mm continuum maps (JCMT and SEST) at $0.45,0.85$, and $1.2 \mathrm{~mm}$ show the presence of a dust core centered at the cluster. With standard assumptions the dust mass is $10 M_{\odot}$; assuming the canonical gas/dust ratio of about 100 , the total core mass is $\sim 1000 M_{\odot}$, which is consistent with what is found from ${ }^{13} \mathrm{CO}$ and $\mathrm{C}^{18} \mathrm{O}$ data. The question now is which mechanism started the star formation process in this cloud, and how it can be sustained over such a long time (i.e., at least 20-25 Myr, the age of a K3 III-star).

Acknowledgements. The James Clerk Maxwell Telescope is operated by the Joint Astronomy Centre on behalf of the Particle Physics and Astronomy Research Council of the United Kingdom, The Netherlands Organisation for Scientific Research, and the National Research Council of Canada. Observations were carried out under projects nls0203, m02bn13, m03bn22, and m05bn27b. This paper is partially based on observations made with the Italian Telescopio Nazionale Galileo (TNG). The TNG is operated on the island of La Palma by the Fundación Galileo Galilei of the INAF (Istituto Nazionale di Astrofisica) at the Spanish Observatorio del Roque de los Muchachos of the Instituto de Astrofisica de Canarias. This research has made use of NASA's Astrophysics Data System Bibliographic Services (ADS) and of the SIMBAD database, operated at CDS, Strasbourg, France. Equatorial coordinates of the NIR-objects were determined using the GAIA software. We thank Robert Zylka for his help with the SIMBA data reduction procedures and scripts, and Giovanna Stirpe for help with the reduction of the spectroscopic data. The spectra were taken at the TNG in Service Mode by Gloria Andreuzzi, to whom we are grateful. We thank Francesco Palla for helpful discussions in Sect. 4. We are indebted to Loris Magnani and Riccardo Cesaroni for commenting on an earlier version of this paper.

\section{References}

Amôres, E. B., \& Lépine, J. R. D. 2005, AJ, 130, 659

Bachiller, R., \& Tafalla, M. 1999, in The Physics of Star Formation and Early Stellar Evolution, NATO Advanced Science Institute, ed. C. J. Lada (Dordrecht: Kluwer)

Basu, S., \& Rana, N. C. 1992, ApJ, 393, 373

Bessell, M. S., \& Brett, J. M. 1988, PASP, 100, 1143

Beuther, H., Schilke, P., Sridharan, T. K., Menten, K. M., \& Wyrowski, F. 2002, A\&A, 383, 892

Blum, R. D., Daminelli, A., \& Conti, P. S. 2001, AJ, 121, 3149

Bohlin, R. C., Savage, B. D., \& Drake, J. F. 1978, ApJ, 224, 132

Brand, J., \& Blitz, L. 1993, A\&A, 275, 67

Brand, J., Wouterloot, J. G. A. 1994, A\&AS, 103, 503 (BW94)

Brand, J., \& Wouterloot, J. G. A. 1995, A\&A, 303, 851

Brand, J., \& Wouterloot, J. G. A. 1998, A\&A, 337, 539

Brand, J., Wouterloot, J. G. A., Rudolph, A. L., \& de Geus, E. J. 2001, A\&A, 377,644
Carpenter, J. M., Snell, R. L., Schloerb, F. P., \& Skrutskie, M. F. 1993, ApJ, 407, 657

Chini, R., Krügel, E., \& Wargau, W. F. 1992, A\&A, 265, 45

Codella, C., Brand, J., Massi, F., Wouterloot, J. G. A., \& Davis, G. R. 2006, A\&A, 457, 891

Deul, E. 1988, Ph.D. Thesis, Univ. of Leiden, The Netherlands

Dickman, R. L., \& Clemens, D. P. 1983, ApJ, 271, 143

Douglas, J. N., Bash, F. N., Bozyan, F. A., Torrence, G. W., \& Wolfe, C. 1996, AJ, 111, 1945

Fich, M. 1984, in The Outer Galaxy, Lecture Notes in Physics, ed. L. Blitz, \& F. Lockman, 306, 187

Fich, M., \& Blitz, L. 1984, ApJ, 279, 125

Fontani, F., Cesaroni, R., Testi, L., et al. 2004, A\&A, 414, 299

Frerking, M. A., Langer, W. D., \& Wilson, R. W. 1982, ApJ, 262, 590

Gingerich, O., \& Kumar, S. S. 1964, AJ, 69, 139

Gregory, P. C., \& Taylor, A. R. 1986, AJ, 92, 371

Hatchell, J., Fuller, G. A., \& Ladd, E. F. 1999, A\&A, 344, 687

Hirano, N., \& Taniguchi, Y. 2001, ApJ, 550, L219

Holland, W. S., Robson, E. I., Gear, W. K., et al. 1999, MNRAS, 303, 659

Jacoby, G. H., Hunter, D. A., \& Christian, C. A. 1984, ApJS, 56, 257

Jenness, T., \& Lightfoot, J. F. 1998, Starlink User Note, 216 http://www .jach.hawaii.edu/JACpublic/stardocs/ sun216.htx/sun216.html

Knapp, G. R., Tremaine, S. D., \& Gunn, J. E. 1978, AJ, 83, 1585

Kobayashi, N., \& Tokunaga, A. T. 2000, ApJ, 532, 423

Koornneef, J. 1983, A\&A, 128, 84

Kramer, C. 1988, Diplomarbeit, I. Phys. Inst., Univ. Köln

Kramer, C., Stutzki, J., Röhrig, R., \& Corneliussen, U. 1998, A\&A, 329, 249

Kumar, B., Sagar, R., Sanwal, B. B., \& Bessell, M. S. 2004, MNRAS, 353, 991

Lada, C. J. 1985, ARA\&A, Vol. 23, 267

Lada, C. J., \& Adams, F. C. 1992, ApJ, 393, 278

Lada, E. A., Evans, N. J. II, Depoy, D. L., \& Gatley, I. 1991, ApJ, 371, 171

Lada, C. J., Alves, J., \& Lada, E. A. 1996, AJ, 111, 1964

Lada, C. J., Muench, A. A., Haisch, K. E. Jr, et al. 2000, AJ, 120, 3162

MacLaren, I., Richardson, K. M., \& Wolfendale, A. W. 1988, ApJ, 337, 355

McClure-Griffiths, N. M., Dickey, J. M., Gaensler, B. M., \& Green, A. J. 2004, ApJ, 607, L127

Meyer, M., Calvet, N., \& Hillenbrand, L. A. 1997, AJ, 114, 288

Molinari, S., Testi, L., Brand, J., Cesaroni, R., \& Palla, F. 1998, ApJ, 505, L39

Ossenkopf, V., \& Henning, Th. 1994, A\&A, 291, 943

Phillips, T. G., Knapp, G. R., Wannier, P. G., et al. 1981, ApJ, 245, 512

Rieke, G. H., \& Lebofsky, M. J. 1985, ApJ, 288, 618

Rohlfs, K., \& Wilson, T. L. 1996, Tools of Radioastronomy, 2nd Ed. (Berlin: Springer-Verlag)

Rolleston, W. R. J., Smartt, S. J., Dufton, P. L., \& Ryans, R. S. I. 2000, A\&A, 363,537

Rudolph, A. L., Brand, J., de Geus, E. J., \& Wouterloot, J. G. A. 1996, ApJ, 458, 653

Russeil, D. 2003, A\&A, 397, 133

Santos, N. C., Yun, J. L., Clemens, D. P., \& Agostinho, R. J. 2000, ApJ, 540, L87

Schmidt-Kaler, Th. 1982, in Landolt-Börnstein, Vol. VI/2b (Springer)

Snell, R. L., Carpenter, J. M., \& Heyer, M. H. 2002, ApJ, 578, 229

Stahler, S. W., Palla, F., \& Ho, P. T. P. 2000, Protostars and Planets IV, ed. V. Mannings, A. P. Boss, \& S. S. Russell (Univ. of Arizona Press), 327

Tafalla, M., Myers, P. C., Caselli, P., \& Walmsley, C. M. 2004, A\&A, 416, 191

Wilson, T. L., \& Matteucci, F. 1992, A\&AR, 4, 1

Wilson, T. L., \& Rood, R. 1994, ARA\&AR, 32, 191

Wouterloot, J. G. A., \& Brand, J. 1989, A\&AS, 80, 149

Wouterloot, J. G. A., \& Brand, J. 1999, A\&AS, 140, 177

Wouterloot, J. G. A., Brand, J., \& Henkel, C. 1988, A\&A, 191, 323

Wouterloot, J. G. A., Brand, J., Burton, W. B., \& Kwee, K. K. 1990, A\&A, 230, 21

Wouterloot, J. G. A., Brand, J., \& Fiegle, K. 1993, A\&AS, 98, 589

Wouterloot, J. G. A., Fiegle, K., Brand, J., \& Winnewisser, G. 1995, A\&A, 311, 236 (Erratum: 1997, A\&A 319, 360)

Zhang, Q., Hunter, T. R., Brand, J., et al. 2005, ApJ, 625, 864 
J. Brand and J. G. A. Wouterloot: Star cluster at the edge of the Galaxy, Online Material p 1

\section{Online Material}


J. Brand and J. G. A. Wouterloot: Star cluster at the edge of the Galaxy, Online Material p 2

Table 4. NIR photometry data.

\begin{tabular}{|c|c|c|c|c|c|c|c|c|c|c|}
\hline No. & $\begin{array}{c}\alpha(2000) \\
\text { h m s }\end{array}$ & $\begin{array}{c}\delta(2000) \\
\circ^{\prime \prime \prime}\end{array}$ & $K$ & $\sigma_{K}$ & $H$ & $\sigma_{H}$ & $J$ & $\sigma_{J}$ & $H-K$ & $J-H$ \\
\hline 3 & $06: 17: 25.79$ & $+14: 55: 00.1$ & 16.44 & 0.09 & 17.57 & 0.16 & 18.70 & 0.23 & 1.125 & 1.128 \\
\hline 4 & $06: 17: 25.73$ & $+14: 54: 50.9$ & 16.57 & 0.10 & 17.12 & 0.09 & 18.21 & 0.16 & 0.556 & 1.083 \\
\hline 5 & $06: 17: 25.57$ & $+14: 55: 05.4$ & 13.06 & 0.04 & 13.98 & 0.05 & 15.46 & 0.02 & 0.912 & 1.479 \\
\hline 7 & $06: 17: 25.42$ & $+14: 54: 28.7$ & 16.22 & 0.14 & 16.74 & 0.12 & 17.12 & 0.09 & 0.518 & 0.383 \\
\hline 8 & $06: 17: 25.42$ & $+14: 54: 23.8$ & 15.56 & 0.05 & 16.21 & 0.08 & 17.24 & 0.08 & 0.644 & 1.035 \\
\hline 9 & $06: 17: 25.37$ & $+14: 54: 59.7$ & 17.65 & 0.20 & 18.60 & 0.29 & 19.20 & 0.39 & 0.948 & 0.597 \\
\hline 10 & $06: 17: 25.33$ & $+14: 54: 30.7$ & 15.58 & 0.06 & 16.05 & 0.04 & 17.25 & 0.10 & 0.472 & 1.200 \\
\hline 11 & $06: 17: 25.28$ & 1:54:46.9 & 16.80 & 0.22 & 17.88 & 0.23 & 19.03 & 0.36 & 1.072 & 1.154 \\
\hline 12 & $06: 17: 2$ & $54: 49.3$ & & 0.0 & & 0.0 & 16. & 0.05 & 1.032 & 1.307 \\
\hline 13 & $06: 1$ & + & 13.2 & 0.0 & 13.50 & 0.04 & & & 0.253 & 0.277 \\
\hline 14 & & & & & & & & & 0.753 & 1.406 \\
\hline 15 & $06: 1$ & & 1. & 0.0 & & & & & & .694 \\
\hline 16 & $06: 1$ & & 16. & 0.1 & & 0.1 & 7 & & 045 & .429 \\
\hline 17 & $06: 1^{1}$ & & 13. & 0.06 & 13. & 0.1 & $14 . ?$ & $0 .($ & 0.239 & 0.488 \\
\hline 20 & 06:17: & +14 & 17.1 & 0.20 & 17.69 & 0.1 & 19.15 & 0.4 & 0.525 & 1.457 \\
\hline 21 & $06: 17: 24.95$ & $+14: 54: 26.6$ & 17.3 & 0.21 & 18.16 & 0.19 & 19.12 & 0.3 & 0.859 & 0.967 \\
\hline 23 & $06: 17: 24.84$ & $+14: 54: 36.9$ & 16.62 & 0.12 & 17.34 & 0.09 & 18.69 & 0.24 & 0.715 & 1.346 \\
\hline 24 & $06: 17: 24.81$ & $+14: 55: 18.7$ & 14.66 & 0.04 & 15.13 & 0.03 & 16.28 & 0.06 & 0.469 & 1.153 \\
\hline 25 & $06: 17: 24.78$ & $+14: 54: 55.9$ & 15.81 & 0.07 & 16.63 & 0.06 & 17.97 & 0.12 & 0.825 & 1.334 \\
\hline 26 & $06: 17: 24.78$ & $+14: 54: 53.8$ & 14.58 & 0.03 & 15.44 & 0.04 & 16.91 & 0.0 & 0.855 & 1.468 \\
\hline 27 & $06: 17: 24.75$ & $+14: 54: 59.1$ & 15.20 & 0.07 & 15.93 & 0.0 & 16.93 & 0.09 & 0.732 & 1.002 \\
\hline 28 & $06: 17: 24.78$ & $+14: 54: 32.5$ & 17.09 & 0.15 & 17.48 & 0.10 & 18.38 & 0.1 & 0.392 & 0.898 \\
\hline 29 & $06: 1$ & & $17 .($ & 0.1 & & 0.1 & 18. & & 706 & 0.949 \\
\hline 30 & $06: 1$ & & & 0.1 & & 0.09 & & 0.16 & 0.348 & 0.858 \\
\hline 31 & & & & 0.05 & 15.64 & 0.05 & 17.02 & 0.06 & 0.770 & 1.382 \\
\hline 33 & $06: 17: 24.33$ & & & 0.04 & & & & & 0.476 & 0.987 \\
\hline 34 & $06: 17: 24.36$ & $+14: 54: 28.5$ & 15.8 & 0.10 & 16.31 & 0.08 & 17.34 & 0.10 & 0.477 & 1.026 \\
\hline 35 & $06: 17: 24.30$ & $+14: 54: 48.2$ & 14.5 & 0.05 & 15.69 & 0.06 & 17.28 & 0.09 & 1.120 & 1.583 \\
\hline 36 & $06: 17: 24.28$ & $+14: 54: 53.0$ & 16.6 & 0.14 & 17.40 & 0.11 & 19.17 & 0.34 & 0.769 & 1.769 \\
\hline 37 & $06: 17: 24.24$ & $+14: 54: 33.1$ & 13.65 & 0.05 & 14.44 & 0.04 & 15.74 & 0.04 & 0.786 & 1.301 \\
\hline 40 & $06: 17: 23.96$ & $+14: 54: 32.5$ & 13.14 & 0.13 & 13.56 & 0.05 & 14.12 & 0.01 & 0.422 & 0.560 \\
\hline 42 & $06: 17: 23.85$ & $+14: 55: 12.9$ & 16.7 & 0.11 & 17.56 & 0.12 & 18.76 & 0.24 & 0.819 & 1.207 \\
\hline 44 & $06: 17: 23.76$ & $+14: 55: 17.8$ & 13.8 & 0.0 & 14.45 & 0.09 & 15.58 & 0.07 & 0.654 & 1.126 \\
\hline 45 & $06: 17: 23.78$ & $+14: 54: 34.0$ & 13.94 & 0.18 & 14.66 & 0.20 & 15.99 & 0.05 & 0.713 & 1.334 \\
\hline
\end{tabular}


J. Brand and J. G. A. Wouterloot: Star cluster at the edge of the Galaxy, Online Material p 3

Table 4. continued.

\begin{tabular}{|c|c|c|c|c|c|c|c|c|c|c|}
\hline No. & $\begin{array}{c}\alpha(2000) \\
\text { h m s }\end{array}$ & $\begin{array}{c}\delta(2000) \\
\circ^{\prime}{ }^{\prime \prime}\end{array}$ & $K$ & $\sigma_{K}$ & $H$ & $\sigma_{H}$ & $J$ & $\sigma_{J}$ & $H-K$ & $J-H$ \\
\hline 46 & $06: 17: 23.79$ & $+14: 54: 27.9$ & 16.25 & 0.10 & 16.74 & 0.08 & 17.78 & 0.12 & 0.487 & 1.037 \\
\hline 47 & & & & & & & 17.45 & & & 1.190 \\
\hline 48 & 3.56 & & .32 & 0.16 & 17. & 12 & 1 & & 378 & 0.978 \\
\hline 49 & $06: 17: 23.56$ & $+14: 54:$ & 17.06 & 0.16 & 18.32 & 0.21 & 19.60 & 2 & 269 & 1.280 \\
\hline 50 & $06: 17: 23.54$ & $+14: 54: 5$ & 16.27 & 0.08 & 16.96 & 0.09 & 18.12 & & .691 & 1.157 \\
\hline 51 & $06: 17: 23.53$ & $+14: 54: 39.6$ & 16.15 & 0.08 & 16.50 & 0.09 & 17.71 & 0 . & .348 & 1.212 \\
\hline 53 & $06: 17: 23.46$ & $+14: 55: 06.6$ & 16.96 & 0.13 & 17.39 & 0.12 & 18.83 & 0.2 & 0.433 & 1.433 \\
\hline 54 & $06: 17: 23.42$ & $+14: 54: 40.0$ & 16.19 & 0.07 & 18.29 & 0.26 & 19.34 & 0. & 2.097 & 1.053 \\
\hline 56 & $06: 17: 23.30$ & $+14: 55: 14.9$ & 15.42 & 0.0 & 15.60 & 0.11 & 15.95 & 0. & 0.173 & 0.350 \\
\hline 57 & $06: 17: 2$ & & & 0.09 & & 0.0 & 17.80 & & .605 & 1.057 \\
\hline 60 & $06: 17: 23.23$ & & & 0.08 & & & & & 0.730 & 1.318 \\
\hline 62 & & & & & & & & & 0.544 & 1.224 \\
\hline 63 & & & & & & & & & & 0.762 \\
\hline 64 & & & & & & & & & & 0.614 \\
\hline 65 & & & & & & & & & & 1.130 \\
\hline 66 & & & & & & & & & & 1.006 \\
\hline 68 & 06: & & & 0. & & & & & 472 & 1.110 \\
\hline 69 & $06: 1$ & & & $0 .($ & & & 1 & & .542 & 1.045 \\
\hline 70 & $06: 17$ & $+14: 5$ & 14.99 & 0.0 & 15. & 0.0 & 15.8 & & .201 & 0.693 \\
\hline 71 & 06:17: & +1 & 15.72 & 0.0 & 15.8 & 0.09 & 16.40 & 0. & .166 & 0.512 \\
\hline 72 & 06:17: & +1 & & 0.0 & 16.2 & 0.0 & 17.2 & 0. & 0.435 & 1.052 \\
\hline 76 & $06: 1$ & & & & 16. & 0. & 16. & & .327 & 0.562 \\
\hline 80 & $06: 1$ & +1 & & & 17. & & 18. & & .607 & 0.915 \\
\hline 81 & $06: 1$ & & & & 17. & & 18. & & 1.027 & 0.593 \\
\hline 83 & $06: 17$ : & & 14 & & & & & & & 1.091 \\
\hline 86 & & & & 0.22 & 17.67 & 0.21 & 18.94 & & 0.639 & 1.275 \\
\hline 87 & & & & & & & & & 0.085 & 1.384 \\
\hline 88 & & & & & & & & & & 0.638 \\
\hline 89 & & & & & & & & & & 1.535 \\
\hline 90 & & & & & & & & & & 0.471 \\
\hline 92 & & & & & & & & & & 1.913 \\
\hline 93 & & & & & & & & & & 0.634 \\
\hline 98 & & & & & & & & & 0.541 & 0.723 \\
\hline 99 & $06: 17: 23.73$ & $+14: 54: 34.7$ & 16.59 & 0.25 & 17.46 & 0.32 & 17.43 & 0.18 & 0.869 & -0.033 \\
\hline
\end{tabular}

